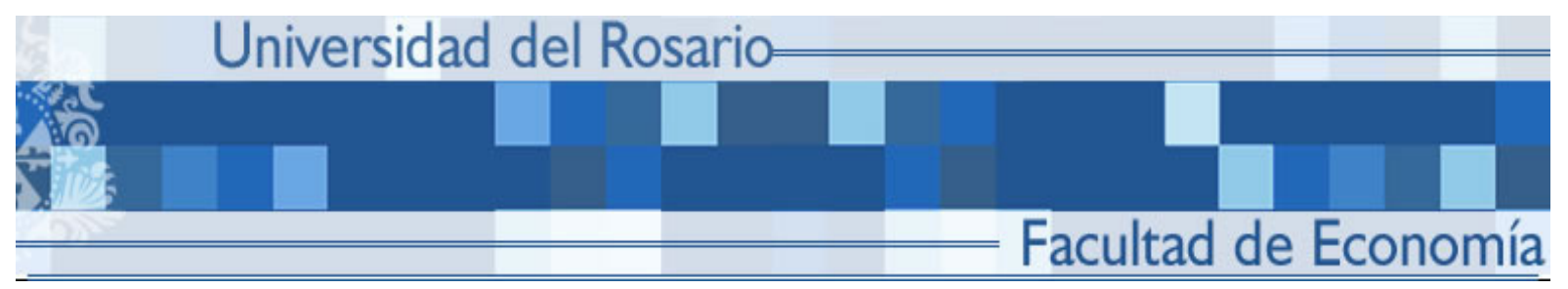

GENETIC TESTING WITH PRIMARY PREVENTION AND MORAL HAZARD

David Bardey

Philippe De Donder

SERIE DOCUMENTOS DE TRABAJO

No. 112

Octubre 2011 


\title{
Genetic testing with primary prevention and moral hazard $^{1}$
}

\author{
David Bardey ${ }^{2}$ and Philippe De Donder ${ }^{3}$
}

October 31, 2011

${ }^{1}$ This research has been undertaken in part while the second author was visiting the University of Rosario. He thanks the university for its generous hospitality. The usual disclaimer applies.

${ }^{2}$ University of Rosario (Colombia) and Toulouse School of Economics (France). Email: david.bardey@gmail.com

${ }^{3}$ Toulouse School of Economics (GREMAQ-CNRS and IDEI), France. Email: dedonder@cict.fr 


\begin{abstract}
We develop a model where a free genetic test reveals whether the individual tested has a low or high probability of developing a disease. A costly prevention effort allows high-risk agents to decrease the probability of developing the disease. Agents are not obliged to take the test, but must disclose its results to insurers. Insurers offer separating contracts which take into account the individual risk, so that taking the test is associated to a discrimination risk.

We study the individual decisions to take the test and to undertake the prevention effort as a function of the effort cost and of its efficiency. We obtain that, if effort is observable by insurers, agents undertake the test only if the effort cost is neither too large nor too low. If the effort cost is not observable by insurers, they face a moral hazard problem which induces them to under-provide insurance. We obtain the counterintuitive result that moral hazard increases the value of the test if the effort cost is low enough. Also, agents may perform the test for lower levels of prevention efficiency when effort is not observable.
\end{abstract}

JEL Codes: D82,G22, I18.

Keywords: discrimination risk, informational value of test, personalized medecine. 


\section{Introduction}

According to Francis S. Collins, director of the U.S. National Institutes of Health, in his book "The language of Life: DNA and the Revolution in Personalized Medicine", there is a revolution currently at play in genetics and medicine. This revolution consists in the increased availability of genetic tests, ever more informative on the underlying health risks of individuals. Collins' book contains a wealth of examples, from mutations in genes known as BRCA1 and BRCA2 ("which increase the lifetime risk of breast cancer to approximately $80 \%$, and of ovarian cancer to about $50 \%$ (page 66$)^{1}$ "), to long QT syndrome ("where individuals who are in the upper end of the QT interval face about a threefold increased risk of sudden death" (page 495)), and to the screening of newborns for 29 different conditions. Collins (2010) contends that "The revolution in human genetics is extending rapidly beyond these less common conditions to reveal the role of individual genetic factors in much more common conditions such as diabetes, heart disease and cancer." (page 1045)", and his book brims with many such examples. For instance, he contends that obesity is "very heritable, with current estimates suggesting that roughly 60 to 70 percent of one's adult body weight is determined by genes. Several of these genes have already been discovered." (page 842).

The main thesis of Collins (2010) (as well as other books, such as Davies (2010)) is that this information is ever more reliable and allows us not only to be better informed about our health risks, but also to use this information to decrease the probability that a given disease will occur in the future. Collins insists that improvement in the assessment of the risk of occurrence of a disease very often allows the individual to take preventive action in order to prevent this disease from occurring. "There are many diseases such as cystic fibrosis or PKU, for which a particular biochemical or DNA test result makes a very strong prediction about the likelihood of illness, and interventions are available" (page 802). There is actually a whole range of such prevention activities: "institution of drug therapies; (...) special diets; (...) surgery or other options" (page 815). As he writes quoting a patient "I know early in my life something I am substantially predisposed to. I now have the opportunity to adjust my life to reduce those odds" (page 1070).

Finally, Collins anticipates that such tests will become ever cheaper in the next years: "As the cost for sequencing the entire genome progressively fall, probably to less than $\$ 1,000$ in the next five or seven years" (page 842 ). Actually, "The $\$ 1,000$ genome" is the title of Davies (2010)'s book.

The objective of our paper is to try and assess the impact of offering a free (genetic) test to individuals on both the private health insurance market and on the welfare of individuals. More precisely, we aim at understanding under what circumstances such a test would be voluntarily taken by individuals, what the consequences of the availability

\footnotetext{
${ }^{1}$ Page numbers refer to the kindle edition of the book.
} 
of testing would be on the extent to which individuals undertake prevention efforts, and whether such a test would increase individual welfare. The simple model we develop to answer these questions has the main ingredients of Collins's story. Agents differ in their risk to develop a disease, with two types ( $L$ and $H$ ) corresponding to two levels of risk in the general population: a fraction $\lambda$ has the high probability $p_{H}$ of developing the disease while the remainder has the low probability $p_{L}<p_{H}$. People are born uninformed about their individual risk level, but can undertake a (genetic or otherwise $^{2}$ ) test in order to assess (without any error) whether they are of a low or high type (in the former case, we talk about a negative test, versus a positive test in the latter case). After the testing phase, agents decide whether to undertake a prevention effort, at a cost, in order to decrease the probability of occurrence of the disease. That is, we model primary prevention (as opposed to secondary prevention, which does not affect the probability that the disease occurs, but decreases its severity). ${ }^{3}$ Collins (2010) provides many examples of both primary and secondary prevention ("discoveries are providing powerful new insights into both treatment and prevention", page 1084).

We assume that prevention is efficient at reducing the risk of illness only if the individual's test is positive (i.e., if he is of a high type). One can give several examples of tests/illnesses with such features, ranging from prophylactic mastectomy in case of mutated BRCA1 gene, to "intense medical surveillance and removal of polyps (that) can be lifesaving for those at high risk" of colon cancer (page 1853). One reason why prevention effort may be efficient only if an individual has a high type is that "it is a combination of the genes that you have inherited and the environment that you live in that determines the outcome. Hence the common saying, "genes load the gun, and environment pulls the trigger" (page 1098). For instance, "Participants in the lifestyle intervention group reduced their risk of developing full-blown diabetes by 58 percent." (page 1313). For macular degeneration, "it became clear that almost 80 percent of the risk could be inferred from a combination of (...) two genetic risk factors, combined with just two environmental risk factors (smoking and obesity)" (page1169). Another reason why effort may be efficient for high risk only is that it has to combine several approaches, including drug therapies: "In many instances, dietary modification turns out to be insufficient (...) Thus drugs in the class known as statins have become the most widely prescribed in the developed world" (page 1313).

We assume perfect competition between profit-maximizing insurers, who observe whether individuals have taken the test, and the result of the test. On the other hand, insurers cannot force individuals to undertake the test, and/or the prevention effort. This corresponds to the situation labeled "disclosure duty" by Barigozzi and Henriet

\footnotetext{
${ }^{2}$ Alternatively, the "test" could be an exploration of family history, which Collins (2010, page 1084) indeed dubs a "free genetic test".

${ }^{3}$ We thus do not cover the illnesses that are entirely driven by genetic conditions and/or for which there is no known prevention effort (such as, for instance, Huntington desease).
} 
(2011), and to the legal environment in New Zealand and the United Kingdom. We also assume, in line with existing conditions, that risk discrimination insurance is not available in the market. Taking the test then corresponds to a lottery, since it means (under disclosure duty and with separating insurance contracts) that the agent ends up with probability $\lambda$ with the contract designed for high types, and with probability $1-\lambda$ with the contract designed for the low type, rather than with the contract designed for uninformed agents and based on the average risk $\lambda p_{H}+(1-\lambda) p_{L}$. In other words, taking the test means supporting a discrimination risk. We already know from previous literature (Hirshleifer, 1971) that, in a classical von Neumann-Morgenstein expected utility framework, risk averse agents will not undertake the test in the simple setting where the test does not allow to better calibrate prevention efforts.

We then add the possibility for the individuals to exert some primary prevention effort in order to decrease their probability of bad health from $p_{H}$ to the lower $p_{H}^{1}{ }^{4}$ The availability of a prevention strategy should give stronger incentives to undertake the test. Whether individuals make the prevention effort and thus decrease their risk is also of interest to the insurers. An open question is whether this prevention effort is observable by insurers. Prevention is easily observable when it takes the form of surgery, or even drug therapy. It is much more difficult to observe if it consists of lifestyle changes such as dietary modifications or exercise. We thus cover the two cases, treating first the situation where the prevention is observable, and then the case where it is not observable by insurers. Throughout our analysis, we stress two dimensions of the prevention effort: its cost for the agent, and its effectiveness, i.e. the amount by which it reduces the risk of someone whose test is positive.

We first study the benchmark situation where the effort is observable, verifiable and contractible by the insurers. Even in this simple situation, our results reveal that the value of information given by the test has an interesting relationship with the cost of the preventive actions. More precisely, we first point out that the genetic test generates a valuable information only for intermediate levels of the prevention cost. When the prevention effort cost is low, even uninformed people (who do not take the test) make the prevention effort, although it is efficient only with probability $\lambda$. In such a case, the genetic test precisely allows to forego the effort (and its cost) if the test is revealed negative. The value of the test, defined as the difference in ex ante utility between taking the test or not, is then increasing with the effort cost, and may become positive if both the cost and efficiency of effort are not too low. For intermediate values of the effort cost, agents undertake the prevention effort only if they have a positive test. ${ }^{5}$ The

\footnotetext{
${ }^{4}$ See Barigozzi and Henriet (2011) for a comparison of legal environments in a setting with observable secondary prevention.

${ }^{5}$ This corresponds to the following two observations by Collins (2010): "Information about an elevated genetic risk may cause people to take actions they otherwise would have ignored" (page 1313), and "She was aware that she was following diet and exercise routines that she probably should have
} 
test then allows them to undertake the prevention effort, and the value of the test is decreasing in the effort cost. This value if positive provided that the effort cost is not too large. Finally, when the effort cost is large, even high type agents do not undertake the effort, and the value of the test is always negative since the only impact of taking the test is to expose agents to the discrimination risk. As is intuitive, the value of the test increases with the efficiency of the prevention effort.

We then turn to the case where effort is not observable by insurers. In order to induce policyholders to undertake effort, they have to provide partial insurance to agents who pretend to undertake the effort. If they were to provide full insurance, as in the previous case, then agents would pretend to undertake effort but would not do it, saving themselves the effort cost. In other words, we are facing a moral hazard problem, solved by insurers by under-providing insurance. A naïve intuition would suggest that this under-provision, by reducing the utility level with effort (compared to the perfect information case) is detrimental to the value of the test, whose only raison d'être is to provide information allowing to calibrate the prevention effort to one's own circumstances. We show that this intuition does not hold in general. More precisely, this intuition is correct for the middle range of values of the effort cost, where the effort is undertaken only in the case of a (positive) test. But it does not hold when the effort cost is low enough that prevention is undertaken both if uninformed or if tested positive. In that case, we show that the value of the test is actually larger with than without moral hazard, because moral hazard degrades more the utility when the test is not taken (and effort is undertaken) than when it is taken (and effort undertaken only in the case of a positive test). Roughly, this is true because insurers have to ration coverage more to uninformed types than to high types in order to induce them to undertake the effort.

Comparing further the cases with and without moral hazard, we obtain two main results. First, for a given efficiency level of prevention, the interval of (intermediate) values of the effort cost which are inducing agents to take the test (i.e., for which the value of the test is positive) moves to the left as we introduce moral hazard considerations. That is, quite counter intuitively, there exist combinations of effort cost and efficiency such that the genetic test is undertaken if and only if effort is not observable by insurers! Second, we find occurrences where the test is undertaken for lower values of the efficiency of effort when this effort is unobservable than when it is observed by insurers. Both results are due to the fact that the value of the test is larger with than without moral hazard when the effort cost is sufficiently low that even uninformed agents undertake the prevention effort.

Finally, we assess the impact of the various ingredients of our model on ex ante (expected) utility or welfare. We start from the situation where there is no insurance,

adhered to anyway, but she found the additional genetic information helpful in inducing a greater sense of urgency to make these changes" (page 1461). 
no genetic test and no prevention effort available, and we measure the impact on welfare of allowing each of these three ingredients as a function of the prevention effort cost. We also show that moral hazard is always detrimental to both the prevention effort decision and ex ante utility of agents. Observe that, in the light of the results presented above, this is not a foregone conclusion. For certain combinations of prevention effort cost and efficiency, the introduction of moral hazard considerations changes the testing and effort decisions of agents. At first sight, such a change could then be beneficial to the prevention decision and generate a larger welfare for agents if moral hazard were to induce agents to test while uninformed agents do not undertake the effort. We show that this situation never happens because, for moral hazard to induce the test, the effort cost need to be low enough that uninformed agents do undertake prevention.

\subsection{Related literature}

This paper is part of a growing literature dealing with genetic testing and the value of information. The seminal paper by Hirshleifer (1971) has established that, if health risk is exogenously determined (i.e., there is no prevention effort available), the value of the information brought by the test is negative, because individuals are faced with a discrimination risk. Doherty and Thistle (1996) have further shown that the private value of information is non-negative only if insurers cannot observe consumers' information status or if consumers can conceal their informational status. ${ }^{6}$ Several papers have extended this analysis to settings with prevention efforts. ${ }^{7}$ As pointed out in Ehrlich and Becker (1972), preventive actions can be primary or secondary.

Secondary prevention (or self-insurance) is analyzed in Barrigozzi and Henriet (2011) and Crainich (2011). Barrigozzi and Henriet (2011) compare several regulatory approaches used in practice, from laissez-faire to the prohibition of tests. They show that policyholders are better off under a "disclosure duty" regulation, which is the one we study in this paper and where policyholders can not been forced by insurers to undertake the test, but are obliged to disclose its results when known. The superiority of this regulation method is mainly due to the fact that it does not create any adverse selection problem for the insurers, while allowing to use the information provided by the test to self insure against the damage. ${ }^{8}$ Crainich (2011) points out that the consequences of regulating the insurers' access to genetic information crucially depend on the nature of the equilibrium in the health insurance market - whether pooling or separating. Crainich

\footnotetext{
${ }^{6}$ Rees and Apps (2006) study how redistributional policies can counteract the discrimination risk in order to induce all buyers to supply their genetic information to the insurers.

${ }^{7}$ Another way to make testing more agreeable to individuals is to introduce a "repulsion from chance" component to their utility, as in Hoel et al. (2006).

${ }^{8}$ Hoy and Polborn (2000) and Strohmenger and Wambach (2000) also study the impact of genetic tests on the health insurance market in the presence of adverse selection.
} 
(2011) also analyzes conditions to ensure that the genetic insurance market suggested by Tabarrok (1994) induces the optimal level of secondary prevention.

Primary prevention is considered in Doherthy and Posey (1998) and Hoel and Iversen (2002). Both papers assume that policyholders are not required to inform insurers about their test results and thus focus on the interplay between risk discrimination and adverse selection. Our framework is closer to Hoel and Iversen (2002). We share the assumption that only high risk people can reduce their health risk thanks to primary prevention actions, but we differ when they assume that uninformed policyholders never undertake preventions while we explore all cases in our paper. Also, Hoel and Iversen (2002) allow for both compulsory and voluntary (supplementary) health insurance.

The main difference between this paper and all the articles which introduce prevention (primary or secondary) is that we assume that primary prevention (especially when it consists of lifestyle improvements such as exercising or eating healthy food) is not observable by insurers, which gives rise to a moral hazard problem solve by providing partial insurance coverage. ${ }^{9}$

\section{Setting and notation}

The economy is composed of a unitary mass of individuals. Each individual may incur a monetary damage of amount $d$ with some probability. Individuals belong to one of two groups according to their risk: a fraction $\lambda$ of individuals are of type $H$ and have a high probability, $p_{H}^{0}$, of incurring the damage (with $0<\lambda<1$ ), while the remaining fraction $1-\lambda$ is of type $L$ and has a lower probability, $p_{L}$ (with $0<p_{L}<p_{H}^{0}<1$ ). Therefore, the average risk in the society is given by $p_{U}^{0}=\lambda p_{H}^{0}+(1-\lambda) p_{L}$.

Individuals are not aware of the group they belong to (i.e., of their risk level) unless they take a genetic test. ${ }^{10}$ The test is assumed to be costless and perfect, in the sense that it tells the individual who takes it with certainty whether he is of type $L$ or $H$. After having taken this test or not, individuals choose whether to exert some prevention effort. Unlike Barigozzi and Henriet (2011), we consider primary prevention -i.e., an effort which decreases the probability that the damage occurs, but does not decrease the damage when it is occurred. For simplicity reason, we assume that the prevention decision is binary and that the effort cost (normalized to zero if no effort is undertaken) $c$ is measured in utility terms rather than in money. We further assume that prevention

\footnotetext{
${ }^{9}$ A recent exception is the paper by Filipova and Hoy (2009), which focuses on surveillance and more precisely on the moral hazard risk of over-consumption of surveillance when financial costs are absorbed by the insurance pool. Also, they concentrate on the consequences of information on prevention, while we endogenize both the prevention and testing decisions.

${ }^{10}$ To shorten the text, we sometimes write that an individual is of type $U$ when he is uninformed about his own type and thus believes that he has type $H$ with probability $\lambda$ and type $L$ with probability $1-\lambda$.
} 
has no effect for a low risk individual, while it decreases the risk of a high risk individual to $p_{H}^{1}$, with $p_{L} \leq p_{H}^{1}<p_{H}^{0}$. We capture the prevention efficiency through $\Delta$ with $\Delta=p_{H}^{0}-p_{H}^{1}$. The parameter $\Delta$ can take any value between zero (prevention has no impact on risk, $p_{H}^{1}=p_{H}^{0}$ ) and $\bar{\Delta}=p_{H}^{0}-p_{L}$ (prevention decreases the risk of a type $H$ agent to the level of a low risk agent, $p_{H}^{1}=p_{L}$ ). The two characteristics of the prevention technology, its cost $c$ and efficiency $\Delta$, will play an important role in our analysis.

We now come to the description of the insurance market. We assume that there is a competitive fringe of profit-maximizing insurers. Insurers offer contracts that are composed of a premium $\pi$ to be paid before the risk realization, and of an indemnity (net of the premium) $I$ paid to the individual once and if the risk has materialized. Contracts can of course be conditioned upon what the insurers observe. We assume that all insurers observe the same elements, so that the competitive pressure results in actuarially fair contracts. Contracts are offered and bought after the individuals have obtained information from the test (provided they chose to take it), but before they exert any prevention effort.

The timing of the model consists in four sequential stages: (1) insurers offer contracts, (2) agents decide whether to take the test or not, (3) they choose one insurance contract (or remain uninsured), and (4) they then exert or nor some prevention effort.

In the rest of the paper, we compute and compare the equilibrium allocations depending upon what is observed by the insurers. Section 3 studies the simplest scenario, where the insurers observe both whether an individual has taken the test or not, the result of the test, and whether the individual exerts a prevention effort or not. Effort is both observable, verifiable and contractible, so that insurers are allowed to condition the contract they offer on both the test result (when one is taken) and the prevention

effort. Section 4 assumes that effort is not observable or contractible, so that insurers face a moral hazard problem.

\section{Perfect information}

In this section, insurers can observe all relevant information. This allows them to condition the contracts they offer on whether a test has been taken, its results and whether effort is provided or not. We then start by describing the contracts offered by the insurers, and we then move to the individuals' decisions.

\subsection{Contracts offered by the insurers}

In order to decide whether to take the test, and whether to exert effort, individuals have to anticipate the contract that will be offered to them by insurers at the last stage of the game. These contracts are conditioned on both the intrinsic risk of the individual (low, 
high or average if the individual has not taken the test) and on whether the individual exerts effort. By assumption, prevention has costs but no benefit when the individual is revealed by the test to be of a low type, so that the contracts offered to type $L$ agents entail no prevention effort. Competition forces insurers to offer actuarially fair contracts, so that individuals prefer full insurance at these actuarially fair terms. Insurers then offer 5 types of contracts.

The first contract is destined to the low type agents (i.e., those who have taken a test whose result has been negative, and who thus exert no effort): the premium is denoted by $\pi_{L}$ and the indemnity (net of the premium) by $I_{L}$. The zero-profitability constraint together with full coverage impose that

$$
\begin{aligned}
\pi_{L} & =p_{L} d, \\
I_{L} & =\left(1-p_{L}\right) d .
\end{aligned}
$$

The ex ante utility of a low type agent buying this contract is then given by

$$
\begin{aligned}
U_{L} & =\left(1-p_{L}\right) v\left(y-\pi_{L}\right)+p_{L} v\left(y-d+I_{L}\right) \\
& =v\left(y-p_{L} d\right) \\
& \equiv v\left(c_{L}\right),
\end{aligned}
$$

where $v($.$) is a classical von Neumann Morgenstein utility function with y$ the individual's exogenous income. We then denote by $c_{L}$ the consumption level of a low type agent.

The second contract will be sold to the high type agent who is not exerting any effort. The same analysis as above results in

$$
\begin{aligned}
\pi_{H}^{0} & =p_{H}^{0} d, \\
I_{H}^{0} & =\left(1-p_{H}^{0}\right) d,
\end{aligned}
$$

and in an individual's utility of

$$
\begin{aligned}
U_{H}^{0} & =\left(1-p_{H}^{0}\right) v\left(y-\pi_{H}^{0}\right)+p_{H}^{0} v\left(y-d+I_{H}^{0}\right) \\
& =v\left(y-p_{H}^{0} d\right) \\
& \equiv v\left(c_{H}^{0}\right),
\end{aligned}
$$

where the superscript 0 indicates that the agent makes no effort.

The third contract is aimed at the high type agent who is exerting effort. We then obtain that

$$
\begin{aligned}
\pi_{H}^{1} & =p_{H}^{1} d \\
I_{H}^{1} & =\left(1-p_{H}^{1}\right) d,
\end{aligned}
$$


with a resulting individual utility of

$$
\begin{aligned}
U_{H}^{1} & =\left(1-p_{H}^{1}\right) v\left(y-\pi_{H}^{1}\right)+p_{H}^{1} v\left(y-d+I_{H}^{1}\right)-c \\
& =v\left(y-p_{H}^{1} d\right)-c \\
& \equiv v\left(c_{H}^{1}\right)-c
\end{aligned}
$$

where the superscript 1 indicates that the agent makes a prevention effort. Observe that the two differences between $U_{H}^{1}$ and $U_{H}^{0}$ are the lower risk (recall that $p_{H}^{1} \leq p_{H}^{0}$ ) and the utility cost of effort $c$.

Insurers also devise contracts to be sold to agents who are not taking the test and not exerting any effort. The risk level of these agents is given by

$$
p_{U}^{0}=\lambda p_{H}^{0}+(1-\lambda) p_{L},
$$

so that they are offered a contract with

$$
\begin{aligned}
\pi_{U}^{0} & =p_{U}^{0} d, \\
I_{U}^{0} & =\left(1-p_{U}^{0}\right) d,
\end{aligned}
$$

which results in an individual's utility level of

$$
\begin{aligned}
U_{U}^{0} & =\left(1-p_{U}^{0}\right) v\left(y-\pi_{U}^{0}\right)+p_{U}^{0} v\left(y-d+I_{U}^{0}\right) \\
& =v\left(y-p_{U}^{0} d\right) \\
& \equiv v\left(c_{U}^{0}\right) .
\end{aligned}
$$

Finally, the fifth contract is devised for the agent who is not taking the test but is exerting effort. The risk of this agent is given by

$$
p_{U}^{1}=\lambda p_{H}^{1}+(1-\lambda) p_{L},
$$

so that he is offered a contract with

$$
\begin{aligned}
\pi_{U}^{1} & =p_{U}^{1} d, \\
I_{U}^{1} & =\left(1-p_{U}^{1}\right) d,
\end{aligned}
$$

and a corresponding individual's utility of

$$
\begin{aligned}
U_{U}^{1} & =\left(1-p_{U}^{1}\right) v\left(y-\pi_{U}^{1}\right)+p_{U}^{1} v\left(y-d+I_{U}^{1}\right)-c \\
& =v\left(y-p_{U}^{1} d\right)-c \\
& \equiv v\left(c_{U}^{1}\right)-c .
\end{aligned}
$$

We now turn to the contract chosen by the agent, i.e. whether they take the test and perform some prevention. We first look at the type of contract (with or without prevention effort) chosen by individuals as a function of whether they have taken the test or not. We then study the test decision in the next section. 


\subsection{The choice of prevention}

We first look at agents who have taken the test in the first stage of the game. These agents know with certainty (since the test is always correct) whether they are of type $L$ (negative test) or $H$ (positive test). Agents of type $L$ have no incentive to perform the effort and so buy the contract $\left(\pi_{L}, I_{L}\right)$ giving them a utility level of $U_{L} \cdot{ }^{11}$ Agents of type $H$ have the choice between two contracts (with and without effort) and choose the contract they prefer by comparing the utility level attained under the two contracts. Then, they buy the contract with effort provided that

$$
\begin{aligned}
& U_{H}^{1}>U_{H}^{0} \\
\Leftrightarrow & v\left(c_{H}^{1}\right)-c>v\left(c_{H}^{0}\right) \\
\Leftrightarrow & c<c_{\max }=v\left(c_{H}^{1}\right)-v\left(c_{H}^{0}\right) .
\end{aligned}
$$

Not surprisingly, this condition imposes an upperbound on the cost of effort. Observe that, if this condition is satisfied, then no insurance firm will propose the no-effort contract $\left(\pi_{H}^{0}, I_{H}^{0}\right)$ at equilibrium. If one firm were to do so, then another firm would propose the effort contract $\left(\pi_{H}^{1}, I_{H}^{1}-\varepsilon\right)$ with $\varepsilon$ small, would attract the patronage of all $H$ type, and would make a strictly positive profit.

We now look at agents who have decided not to take the test. These agents do not know their true type, but only that they are of average type $U$. They choose the contract specifying effort offered by insurers to type $U$ if it gives them a higher utility level than the same contract without prevention-i.e. if

$$
\begin{aligned}
& U_{U}^{1}>U_{U}^{0}, \\
\Leftrightarrow & v\left(c_{U}^{1}\right)-c>v\left(c_{U}^{0}\right) \\
\Leftrightarrow & c<c_{\min }=v\left(c_{U}^{1}\right)-v\left(c_{U}^{0}\right) .
\end{aligned}
$$

We can apply the same reasoning as above to show that, if it is individually optimal for an individual who has not taken the test to make a protection effort (resp., not to make an effort), then only the corresponding contract $\left(\pi_{U}^{1}, I_{U}^{1}\right)$ (resp., the contract $\left.\left(\pi_{U}^{0}, I_{U}^{0}\right)\right)$ will be offered at equilibrium by private firms to this individual.

We first show that the threshold prevention cost level $c_{\min }$ below which of average type $U$ choose a contract with effort is lower than the threshold cost level $c_{\text {max }}$ below which agents of high type do some prevention.

Result $1 c_{\min }<c_{\max }$ if $\Delta>0$, while $c_{\min }=c_{\max }=0$ if $\Delta=0$.

\footnotetext{
${ }^{11}$ It is straightforward that agents prefer to be fully insured at an actuarially fair rate rather than not buying any contract and shouldering their risk alone.
} 
Proof. First, note that $c_{H}^{1}-c_{H}^{0}=\left(p_{H}^{0}-p_{H}^{1}\right) d$ while $c_{U}^{1}-c_{U}^{0}=\lambda\left(p_{H}^{0}-p_{H}^{1}\right) d$. We then have that $c_{\min }=c_{\max }=0$ if $\Delta=0$. If $\Delta>0, c_{H}^{1}-c_{H}^{0}>c_{U}^{1}-c_{U}^{0}=\lambda\left(p_{H}^{0}-p_{H}^{1}\right) d$. Moreover, as $c_{U}^{1}>c_{H}^{1}$ and $c_{U}^{0}>c_{H}^{0}$, the concavity of the function $v($.$) implies that$ $c_{\min }<c_{\max }$.

As the cost of effort does not depend on the type, it is always effective if type $H$, but not always effective if type $U$. Moreover, due to the higher actuarial premium paid by policyholders of type $H$, they are characterized by a lower consumption, so that their marginal utility is higher. They thus gain more than average type $U$ from the lower premium made possible by the prevention effort.

Finally, it is easy to see that, if condition (1) is not satisfied, then no agent chooses to exert effort at equilibrium, and our model boils down to a special case of Hoel et al. (2002).

We then have the following result:

Result 2 Depending on the cost of prevention $c$, we are in one of the following three cases:

a) $c<c_{\text {min }}$ : all individuals who have chosen not to take the test buy a contract prescribing prevention effort, as well as agents who have taken a test and discovered that they belong to the high risk type.

b) $c_{\min }<c<c_{\max }$ : only individuals who have taken the test and who are of a high risk type do buy a contract prescribing prevention.

c) $c>c_{\max }$ : no one buys a contract with prevention.

We now look at how these two threshold costs are affected by variations in the effectiveness of prevention. ${ }^{12}$

Result 3 a) Both $c_{\min }$ and $c_{\max }$ increase in $\Delta$.

b) The distance between $c_{\max }$ and $c_{\min }$ increases in $\Delta$.

b)

Proof. a) $\partial c_{\min } / \partial \Delta=\lambda d v^{\prime}\left(c_{U}^{1}\right)>0$ and $\partial c_{\max } / \partial \Delta=d v^{\prime}\left(c_{H}^{1}\right)>0$.

$$
\frac{\partial\left(c_{\max }-c_{\min }\right)}{\partial \Delta}=d\left[v^{\prime}\left(c_{H}^{1}\right)-\lambda v^{\prime}\left(c_{U}^{1}\right)\right]>0
$$

A higher effectiveness of prevention allows to decrease the premium asked by insurers for the contracts prescribing effort. Agents are then accepting these contracts for larger values of the utility cost of effort, explaining why both $c_{\min }$ and $c_{\max }$ increase with $\Delta$.

\footnotetext{
${ }^{12}$ Throughout the paper, when varying $\Delta$, we keep $p_{H}^{0}$ fixed and we decrease $p_{H}^{1}$. In other words, we replace $p_{H}^{1}$ by $p_{H}^{0}-\Delta$ throughout the paper. Also, to simplify notation, we write $c_{\min }$ and $c_{\max }$ rather than $c_{\min }(\Delta)$ and $c_{\max }(\Delta)$.
} 
The second part of the result shows that the maximum cost compatible with an agent of type $H$ making a prevention effort increases more rapidly with $\Delta$ than the maximum cost for which an agent uninformed about his type undertakes prevention. This is due to two factors. First, type $H$ has a higher marginal utility than type $U$ (because he pays a larger premium). Second, the prevention effort always decreases the risk for type $H$, while it is effective only with probability $\lambda$ for someone knowing only his average type. Finally, when $\Delta$ increases, the length of the interval of cost values compatible with type $H$ agents also increases (i.e., $c_{\max }-c_{\min }$ increases).

We now move to the first stage of the model, and assess under what circumstances individuals choose to make the test.

\subsection{To test or not to test}

To solve the first stage decision of the individual i.e. whether taking the test is worth its while, we have to make an assumption on the value of $c$, since it determines under what circumstances an individual makes a prevention effort. We will cover the three cases: $c>c_{\max }$ (so that effort is never undertaken), $c<c_{\min }$ (so that effort is always undertaken, except if a test is taken and results in a low type), and finally $c_{\min }<c<$ $c_{\max }$ (where the effort is undertaken only in the case of a positive test).

In all cases, we define as the value of the test, denoted by $\Psi(c, \Delta)$, the difference between the utility the agent gets with and without taking the test (anticipating in both cases the contract he will buy and whether he will make the prevention effort). Recall that the individual takes the test if and only if this value is positive.

\subsubsection{No one undertakes prevention: $c \geq c_{\max }$}

Result 4 When $c \geq c_{\max }, \Psi(c, \Delta)<0, \forall(c, \Delta)$ so that the test is not taken.

Proof. In that case, an individual decides to take the test if

$$
\begin{aligned}
& \Psi(c, \Delta)=\lambda U_{H}^{0}+(1-\lambda) U_{L}-U_{U}^{0}>0 \\
& \Leftrightarrow \quad \lambda v\left(y-p_{H}^{0} d\right)+(1-\lambda) v\left(y-p_{L} d\right)-v\left(y-p_{U}^{0} d\right)>0,
\end{aligned}
$$

which is never true.

This is the well known (Hirshleifer, 1971) result of the negative value of a genetic test whose results are observable and contractible but which does not allow the individual to use the information to mitigate his risk. The intuition is that taking the test is like buying a lottery, with a good outcome with probability $1-\lambda$ and a bad outcome with probability $\lambda$. On the other hand, by not taking the test, the individual obtains a certain payoff (since he is perfectly insured) at an actuarially fair rate. If the individual is risk averse i.e. exhibits a concave utility function $v($.$) (in the expected utility framework),$ 
he prefers the sure and actuarially fair payoff to the lottery. We call this drawback of the test the discrimination risk, in line with Barigozzi and Henriet (2011).

Observe that $\Psi$ is independent of both the cost and effectiveness of prevention, as long as the cost $c$ is larger than the threshold $c_{\max }$ (which increases with $\Delta$ ). We then have that $\Psi(c, \Delta) \equiv \Psi_{0}<0$ for $c>c_{\max }$.

We now move to the case where effort is undertaken even when the test is not taken.

\subsubsection{Uninformed types undertake prevention: $c \leq c_{\min }$}

The value of the test is given by

$$
\begin{aligned}
\Psi(c, \Delta) & =\lambda U_{H}^{1}+(1-\lambda) U_{L}-U_{U}^{1} \\
& =\lambda\left(v\left(y-p_{H}^{1} d\right)-c\right)+(1-\lambda) v\left(y-p_{L} d\right)-\left(v\left(y-p_{U}^{1} d\right)-c\right) \\
& =(1-\lambda) c-\left[v\left(y-p_{U}^{1} d\right)-\left(\lambda v\left(y-p_{H}^{1} d\right)+(1-\lambda) v\left(y-p_{L} d\right)\right)\right] .
\end{aligned}
$$

The first term in (3) measures the gain from the test, which allows to forgo the prevention effort cost $c$ if the test proves negative (i.e., with probability $1-\lambda$ ) while the terms between brackets represent the drawback from taking the test (moving from a certain payoff to a lottery with the same average payoff, since effort is undertaken even if the test is not taken, but pays off only if the agent has a high risk).

The following result summarizes how the cost and efficiency of effort impact the value of the test when $c \leq c_{\min }$.

Result 5 a) When $c \leq c_{\text {min }}$, we have that

$$
\begin{aligned}
& \frac{\partial \Psi(c, \Delta)}{\partial c}=1-\lambda>0, \\
& \frac{\partial \Psi(c, \Delta)}{\partial \Delta}=\lambda d\left[v^{\prime}\left(c_{H}^{1}\right)-v^{\prime}\left(c_{U}^{1}\right)\right]>0 .
\end{aligned}
$$

b) $\Psi(0,0)=\Psi_{0}$ and $\Psi(0, \bar{\Delta})=0$.

Proof. Straightforward differentiation for a) and use of definition of $\Psi(0, \Delta)$ for b).

The result that the value of the test increases with the cost of prevention effort $c$ may seem counter-intuitive, and is due to the fact that the test allows to forgo making the effort when it is negative. The value of the test also increases with the prevention efficiency $\Delta$ : although the expected monetary gain associated to a lower risk after prevention is the same whether the test is taken or not, the marginal utility of money is larger when taking the test, since the gain occurs when the individual pays the large 
premium associated to being of type $H$ rather than the average premium when the test is not taken.

When the cost of effort is nil, we are essentially back to the classical case: there is nothing to be gained by taking the test, since the gain from testing is to save on the cost of effort when the test is negative. If $\Delta=0$, then $\Psi(c, 0)=\Psi_{0}$ since the prevention effort is totally ineffective and never undertaken. We also have that $\Psi(0, \bar{\Delta})=0$ since in that case the costless prevention allows everyone to reduce his risk level to $p_{L}$ whether they take the test or not, and there is no discrimination risk. Observe that a corollary to Result 5 is that $0>\Psi(0, \Delta)>\Psi_{0}$ for all $0<\Delta<\bar{\Delta}$ : the value of the test is negative, but less so than in case 1 (when $c \geq c_{\max }$ ) because, by making the prevention effort when $c=0$, the individual reduces his risk and then also the riskiness of the lottery associated with taking the test $\left(c_{H}^{1}\right.$ is closer to $c_{L}$ than $c_{H}^{0}$ since $p_{H}^{1}$ is lower than $\left.p_{H}^{0}\right)$.

We are now in a position to state the following result (proved in Appendix):

Result 6 When $c \leq c_{\text {min }}$, the value of the test, $\Psi(c, \Delta)$, is positive provided that the prevention effort's cost $c$ and efficiency $\Delta$ are large enough. Formally,

a) there exists a unique value of $\Delta$, denoted by $\tilde{\Delta}$, such that $0<\tilde{\Delta}<\bar{\Delta}$ and $\Psi\left(c_{\min }, \tilde{\Delta}\right)=$ 0 ;

b) for all $\Delta \geq \tilde{\Delta}$, there exists a unique value of $c$, denoted by $\tilde{c}_{1}(\Delta)$, such that $0 \leq$ $\tilde{c}_{1}(\Delta) \leq c_{\min }$ and $\Psi\left(\tilde{c}_{1}(\Delta), \Delta\right)=0$;

c) $\Psi(c, \Delta)>0$ for all $\Delta>\tilde{\Delta}$ and $\tilde{c}_{1}(\Delta)<c<c_{\min }$;

d) for all $\Delta \geq \tilde{\Delta}, \tilde{c}_{1}(\Delta)$ decreases with $\Delta$;

e) $\tilde{c}_{1}(\bar{\Delta})=0$.

The intuition for this result holds as follows. The value of the test is negative if the efficiency of prevention is low: in that case, individuals make an effort only for low values of the prevention cost $c$. But since the gain from taking the test resides in foregoing this cost of effort when the test is negative (see (3)), a low cost of effort means that this gain is too low to compensate for the discrimination risk entailed by the test. If the prevention efficiency $\Delta$ is large enough, agents make an effort even when its cost is large, in which case the gain from the test is also large and more than compensates the discrimination risk. Formally, we identify both a threshold on effort efficiency and cost above which the value of the test is positive. The threshold cost decreases with prevention efficiency: as explained above, the value of the test increases with $\Delta$, so that it remains positive for lower values of $c$ as $\Delta$ increases. When $\Delta$ reaches $\bar{\Delta}$, the value of the test is positive for all values of $c \leq c_{\text {min }}$.

We then move to the intermediate case, where effort is undertaken if and only if the policyholder is $H$. 


\subsubsection{Only informed types undertake prevention: $c_{\min }<c<c_{\max }$}

In such a case, the value of the test for a policyholder is given by

$$
\begin{aligned}
\Psi(c, \Delta) & =\lambda U_{H}^{1}+(1-\lambda) U_{L}-U_{U}^{0}, \\
& =\lambda\left(v\left(y-p_{H}^{1} d\right)-c\right)+(1-\lambda) v\left(y-p_{L} d\right)-v\left(y-p_{U}^{0} d\right) .
\end{aligned}
$$

In this case, taking the test is a necessary condition to make a prevention effort. A positive test (obtained with probability $\lambda$ ) allows to decrease the risk by $\Delta$. The lottery associated with taking the test then has a larger average consumption level than the sure payoff when the test is not taken.

The following result states how the cost and efficiency of effort impact the value of the test when $c_{\min }<c<c_{\max }$.

Result 7 When $c_{\min }<c<c_{\max }$, straightforward differentiation of (6) shows that

$$
\begin{aligned}
& \frac{\partial \Psi(c, \Delta)}{\partial c}=-\lambda<0, \\
& \frac{\partial \Psi(c, \Delta)}{\partial \Delta}=\lambda d v^{\prime}\left(c_{H}^{1}\right)>0 .
\end{aligned}
$$

The value of the test increases with prevention efficiency $\Delta$, but decreases with the cost of effort $c$. This latter result is in stark contrast with the one obtained when even uninformed types undertake prevention, where taking the test allowed not to make the prevention effort in case of a negative result.

We then obtain the following result.

Result 8 When $c_{\min } \leq c<c_{\max }$, the value of the test is positive provided that the prevention efficiency $\Delta$ is large while the effort cost $c$ is small. Formally,

a) for all $\Delta \geq \tilde{\Delta}$ (as defined in Result 6), there exists a unique value of $c$, denoted by $\tilde{c}_{2}(\Delta)$, such that $c_{\min } \leq \tilde{c}_{2}(\Delta)<c_{\max }$ and $\Psi\left(\tilde{c}_{2}(\Delta), \Delta\right)=0$;

b) $\Psi(c, \Delta)>0$ for all $\Delta>\tilde{\Delta}$ and $c_{\min }<c<\tilde{c}_{2}(\Delta)$;

c) for all $\Delta \geq \tilde{\Delta}, \tilde{c}_{2}(\Delta)$ increases with $\Delta$;

d) $\tilde{c}_{1}(\tilde{\Delta})=\tilde{c}_{2}(\tilde{\Delta})=c_{\min }$ and $c_{\min }<\tilde{c}_{2}(\bar{\Delta})<c_{\max }$.

In this case, taking the test allows to make the prevention effort in case the test is positive. It is then intuitive that the value of the test is positive provided that prevention is cost effective -i.e., that the effectiveness of prevention $\Delta$ is large compared to its cost c. More precisely, the effectiveness of prevention has to be larger than the same threshold identified in Result 6 for the value of the test to be positive for low costs of effort in this 
case. As effectiveness increases, the threshold cost below which the value of the test is positive increases, so that the test is undertaken for larger values of $c$.

Figure 1 provides a graphical illustration of the value of the test as a function of prevention cost for four different values of the prevention efficiency. Throughout the paper, graphical illustrations are based on the following assumptions: $v(c)=\sqrt{c}, y=5$, $d=3, \lambda=0.3, p_{L}=0.1, p_{H}^{0}=0.6$, so that $\bar{\Delta}=0.5$.

Insert Figure 1 around here

\subsection{Testing and effort at equilibrium}

We now summarize our results so far in the following proposition.

Proposition 1 a) If the efficiency of prevention is low enough $(\Delta \leq \tilde{\Delta})$, the test is never chosen, whatever the prevention cost.

b) If the efficiency of prevention is large enough $(\Delta>\tilde{\Delta})$, the test is chosen only if the prevention cost takes intermediate values: $\tilde{c}_{1}(\Delta) \leq c \leq \tilde{c}_{2}(\Delta)$.

c) The set of values of the prevention cost compatible with agents taking the test increases with the prevention efficiency.

We already know from Hirshleifer (1971) that the value of the test for agents is negative in the absence of prevention effort. Prevention may increase the value of the test, because the test determines whether prevention has a benefit or not. Hence, a large enough efficiency of prevention is a necessary condition for the test to be taken, as shown in part a) of Proposition 1. Part b) is less intuitive. Recall that if the prevention cost is low $\left(c<c_{\text {min }}\right)$, prevention is undertaken in the absence of test. The gain from taking the test is then that it allows not to do a prevention effort if the test is negative. The test then allows to save the prevention cost $c$ (with probability $1-\lambda$ ). If the prevention cost is too low, then this gain from taking the test is dominated by the lottery exposure such that the value of the test remains negative. If the prevention cost is larger $\left(c_{\min }<c<c_{\max }\right)$, agents undertake prevention only if they obtain a positive test. Taking the test is then a necessary condition to make the prevention effort, and the gain from the test decreases with the cost of prevention. If this cost is too large, the value of the test remains also negative.

The following proposition states when prevention is undertaken as a function of its cost and efficiency. 
Proposition 2 a) If the efficiency of prevention is low enough $(\Delta \leq \tilde{\Delta})$, then all agents undertake prevention if its cost is low enough $\left(c<c_{\text {min }}\right)$ while no one undertakes prevention otherwise (if $c>c_{\min }$ ).

b) If the efficiency of prevention is large enough $(\Delta>\tilde{\Delta})$, then everyone undertakes prevention if its cost is low enough $\left(c<\tilde{c}_{1}(\Delta)\right)$, only people of type $H$ undertake prevention if its cost is intermediate $\left(\tilde{c}_{1}(\Delta) \leq c \leq \tilde{c}_{2}(\Delta)\right)$ while no one makes a prevention effort otherwise (i.e., if $c>\tilde{c}_{2}(\Delta)$ ).

We illustrate the results of Propositions 1 and 2 on Figure 2, which depicts the thresholds $\tilde{c}_{1}$ (in yellow), $c_{\min }$ (in blue), $\tilde{c}_{2}$ (in green) and $c_{\max }$ (in purple) as functions of $\Delta$. With this numerical example, the value of $\tilde{\Delta}$ is $4 \%$. The area between the curves $\tilde{c}_{2}(\Delta)$ and $\tilde{c}_{1}(\Delta)$ represents the combinations of prevention cost and efficiency for which agents take the test, and where they make an effort only if this test is positive. Outside of this region, no individual takes the test. Combinations of $(c, \Delta)$ located below the $c_{\min }$ and $\tilde{c}_{1}(\Delta)$ curves are such that everyone makes the prevention effort, while combinations above the $c_{\min }$ and $\tilde{c}_{2}(\Delta)$ curve are such that no prevention effort is made.

Insert Figure 2 around here

We now move to the case where both the test and its results are observable and contractible, but where the prevention effort is not.

\section{Unobserved prevention effort}

In that case, we have a moral hazard problem, since the desired prevention effort has to be induced by the insurer by adequately crafting the insurance contracts. We proceed as in section 3 and we first study the contracts proposed by the insurers before moving to the choice of prevention effort and of testing by the agents.

\subsection{Contracts offered by the insurers}

First, observe that contracts offered to agents who the insurers do not wish to induce to make a prevention effort are unchanged, compared to the previous section. These are the contracts offered to low-type (for whom making a prevention effort is not worthwhile), $\left(\pi_{L}, I_{L}\right)$, and to the high type $\left(\pi_{H}^{0}, I_{H}^{0}\right)$ and the average type $\left(\pi_{U}^{0}, I_{U}^{0}\right)$ who need not be induced to make an effort. 
Look now at the contract offered to a high type who the insurer would like to induce to make an effort, which we denote by $\left(\pi_{H}^{1}, I_{H}^{1}\right)$. For the individual to make an effort, it must be the case that the following incentive compatibility (IC hereafter) constraint holds:

$$
\left(1-p_{H}^{1}\right) v\left(b_{H}^{1}\right)+p_{H}^{1} v\left(d_{H}^{1}\right)-c \geq\left(1-p_{H}^{0}\right) v\left(b_{H}^{1}\right)+p_{H}^{0} v\left(d_{H}^{1}\right),
$$

where $b_{H}^{1}$ and $d_{H}^{1}$ denote the consumption level of a type $H$ individual buying the $\left(\pi_{H}^{1}, I_{H}^{1}\right)$ contract in case they are lucky and in case the damage occurs-i.e.,

$$
\begin{aligned}
b_{H}^{1} & =y-\pi_{H}^{1}, \\
d_{H}^{1} & =y-d+I_{H}^{1} .
\end{aligned}
$$

The IC constraint (7) states that the individual, when buying the contract $\left(\pi_{H}^{1}, I_{H}^{1}\right)$, is at least as well off making an effort (the LHS of (7)) than pretending to make one (the RHS of (7)). It is straightforward to see that such a result cannot be attained if the individual is provided with full coverage, since in that case consumption levels are equalized across states of the world $\left(b_{H}^{1}=d_{H}^{1}\right)$, and the individual never makes an effort. As pointed out by Shavell (1979), in such a case, the only way for the insurer to induce effort making is then to restrict the coverage offered to the individual (the competition between insurers ensures that the contracts remain actuarially fair). We denote the contracts as

$$
\begin{aligned}
\pi_{H}^{1} & =\alpha_{H} p_{H}^{1} d, \\
I_{H}^{1} & =\alpha_{H}\left(1-p_{H}^{1}\right) d,
\end{aligned}
$$

where $\alpha_{H}$ is the (maximum) coverage rate offered to individuals of type $H$ in order to induce them to make an effort. The value of $\alpha_{H}$ is implicitly obtained by solving the IC constraint (7) with equality. Restated in terms of $c$, we then obtain that

$$
c=\left(p_{H}^{0}-p_{H}^{1}\right)\left(v\left(b_{H}^{1}\right)-v\left(d_{H}^{1}\right)\right) .
$$

The IC constraint (8) equalizes, on its LHS, the cost of effort with its benefit on the RHS, given the contract offered to a high type pretending to undertake prevention. This benefit is the product of the efficiency of the prevention effort, $\Delta$, with the utility gap between the two states of the world (sick or healthy) when making the effort. We have that $b_{H}^{1}>d_{H}^{1}$ : the insured is better off if the damage does not occur, which gives him the exact incentive needed to support the prevention effort cost $c$.

Observe that the same argument as in the previous section explains why the insurers offer either the contract $\left(\pi_{H}^{1}, I_{H}^{1}\right)$ or the contract $\left(\pi_{H}^{0}, I_{H}^{0}\right)$ to individuals of type $H$, depending upon which of the two contracts gives more utility to these buyers. In other words, competition among insurers ensures that only the welfare-maximizing contract (given the observability constraints) is offered to types $H$. 
Insurers face a similar problem with the individuals who have not taken the test. The effort-inducing contract offered to them is $\left(\pi_{U}^{1}, I_{U}^{1}\right)$ with

$$
\begin{aligned}
\pi_{U}^{1} & =\alpha_{U} p_{U}^{1} d \\
I_{U}^{1} & =\alpha_{U}\left(1-p_{U}^{1}\right) d
\end{aligned}
$$

and with $\alpha_{U}$ satisfying the following incentive compatibility constraint

$$
c=\lambda\left(p_{H}^{0}-p_{H}^{1}\right)\left(v\left(b_{U}^{1}\right)-v\left(d_{U}^{1}\right)\right),
$$

with

$$
\begin{aligned}
b_{U}^{1} & =y-\pi_{U}^{1}, \\
d_{U}^{1} & =y-d+I_{U}^{1}
\end{aligned}
$$

and $b_{U}^{1}>d_{U}^{1}$

Result $9 \alpha_{U}<\alpha_{H}<1$.

Proof. Cf. appendix.

There are two effects at play, both pushing towards a larger coverage rate for type $H$ than for type $U$. First, the expected effectiveness of the prevention effort is larger for type $H$ than for an average type, since for the latter there is a probability $1-\lambda$ that his effort is actually worthless. Second, the utility gap between the good and bad states of the world is larger for type $H$ than for type $U$ for a given coverage level, because the insurance premium is larger for $H$ than for $U$. Both effects explain why it is less necessary to underprovide insurance to a type $H$ than to an average type in order to induce them to undertake the costly prevention effort.

We now look at the impact of prevention cost and efficiency on the coverage offered by insurers.

Result 10 a) $\alpha_{H}$ and $\alpha_{U}$ are decreasing in $c$. There exists a maximum value of $c$, denoted by $\bar{c}_{H}$ (respectively, $\bar{c}_{U}$ ) such that effort by type $H$ (resp., $U$ ) may be induced only if $c \leq \bar{c}_{H}$ (resp., $\left.c \leq \bar{c}_{U}\right)$. Moreover, $\bar{c}_{U}<\bar{c}_{H}$.

b) $\alpha_{H}$ and $\alpha_{U}$ are increasing in $\Delta$ if and only if the respective conditions hold:

$$
\begin{aligned}
\alpha_{H} & \leq-\frac{v^{\prime}\left(\chi_{H}\right)}{v^{\prime \prime}\left(\chi_{H}\right)} \frac{1}{\Delta d}, \\
\alpha_{U} & \leq-\frac{v^{\prime}\left(\chi_{U}\right)}{v^{\prime \prime}\left(\chi_{U}\right)} \frac{1}{\Delta d \lambda},
\end{aligned}
$$

with $\chi_{H} \in\left[d_{H}^{1}, b_{H}^{1}\right]$ and $\chi_{U} \in\left[d_{U}^{1}, b_{U}^{1}\right]$ respectively. 
Proof. Cf. appendix

Recall that the amount of coverage offered to type $i \in\{H, U\}$ equalizes the cost and benefit of prevention effort for the corresponding type, given the contract offered to someone promising to make an effort. Recall also that the benefit of prevention is the product of the efficiency of prevention, $\Delta$, by the utility difference between good and bad states of the world when making the effort (see equations (8) and (9)). As the cost of effort increases, it is necessary to increase this utility difference, and hence to reduce the coverage $\alpha_{i}$ offered to an individual of type $i$. There is a maximum value of the effort cost, $\bar{c}_{i}$, such that it is possible to induce agents of type $i$ to undertake the prevention effort by degrading their coverage. This maximum value of $c$ corresponds to $\alpha_{i}=0$. Intuitively, the maximum prevention cost compatible with an agent making an effort is lower for type $U$ (when effort works with probability $\lambda$ ) than for type $H$. As for the benefit of prevention, it need not increase with prevention efficiency, because a larger value of $\Delta$ decreases the utility gap between states of the world for a given coverage level (both consumption levels $b_{i}^{1}$ and $d_{i}^{1}$ increase by the same amount with $\Delta$, but the marginal utility is larger in the bad state of the world -i.e., with $d_{i}^{1}$ ). The coverage rate $\alpha_{i}$ increases with $\Delta$ provided that this effect is smaller than the direct impact of a larger $\Delta$ on the benefit from prevention (first parenthesis in the RHS of (8) and (9)). This is the case if $\Delta, d, \lambda$ (in the case of $i=U$ ) and the individuals' risk aversion are small enough. ${ }^{13}$

Figure 3 illustrates Result 10 for our numerical example.

Insert Figure 3 around here

We now move to the prevention choice of agents.

\subsection{The choice of prevention}

An individual of type $H$ chooses the contract inducing effort (with the expected utility denoted by $U_{H}^{1 M H}$ ) rather than the other one proposed to his type if

$$
\begin{aligned}
& U_{H}^{1 M H}>U_{H}^{0} \\
\Leftrightarrow & \left(1-p_{H}^{1}\right) v\left(b_{H}^{1}\right)+p_{H}^{1} v\left(d_{H}^{1}\right)-c>v\left(c_{H}^{0}\right) \\
\Leftrightarrow & c<c_{\max }^{M H}=\left(1-p_{H}^{1}\right) v\left(b_{H}^{1}\right)+p_{H}^{1} v\left(d_{H}^{1}\right)-v\left(c_{H}^{0}\right) .
\end{aligned}
$$

The following lemma establishes that the threshold $c_{\max }^{M H}$ is low enough that the IC constraint (8) can be satisfied for a positive coverage rate $\alpha_{H}$.

\footnotetext{
${ }^{13}$ The non monotic relationship between the prevention efficiency and the level of coverage in ex ante moral hazard model has already been pointed out in Bardey and Lesur (2005).
} 
Lemma $1 c_{\max }^{M H}<\bar{c}_{H}$.

Proof. When $c=\bar{c}_{H}$, we have $\alpha_{H}=0$ so that the agent is not insured at all (and is indifferent between making the prevention effort or not). His utility is then lower than what he gets under $U_{H}^{0}$, where he is fully insured at an actuarially fair price (without effort). Since $U_{H}^{1 M H}$ is decreasing in $c$ (because of both the direct effect of a higher $c$ and the indirect impact through the decrease in coverage rate) while $U_{H}^{0}$ is not affected by $c$, the claim is proved.

Likewise, the condition under which it is optimal for an individual who has not taken the test to exert some prevention effort is

$$
\begin{aligned}
& U_{U}^{1 M H}>U_{U}^{0}, \\
\Leftrightarrow & \left(1-p_{U}^{1}\right) v\left(b_{U}^{1}\right)+p_{U}^{1} v\left(d_{U}^{1}\right)-c>v\left(c_{U}^{0}\right) \\
\Leftrightarrow & c<c_{\min }^{M H}=\left(1-p_{U}^{1}\right) v\left(b_{U}^{1}\right)+p_{U}^{1} v\left(d_{U}^{1}\right)-v\left(c_{U}^{0}\right) .
\end{aligned}
$$

Similarly to the case of type $H$, the following lemma shows that the threshold $c_{m i n}^{M H}$ is compatible with a positive value of $\alpha_{U}$ :

Lemma $2 c_{\min }^{M H}<\bar{c}_{L}$.

Proof. Similar to the proof of Lemma 1.

Note that, in both cases, we can apply the same reasoning as in the previous section to show that, if it is individually optimal for an individual to make a protection effort (resp., not to make an effort), then only the corresponding contract $\left(\pi_{i}^{1}, I_{i}^{1}\right)$ (resp., the contract $\left.\left(\pi_{i}^{0}, I_{i}^{0}\right)\right)$ will be offered at equilibrium by private firms to this individual $(i=U, H)$.

Result 11 The threshold values of the prevention effort which determine under what circumstances an individual makes a prevention effort decrease when this effort is not observable by the insurers: $c_{\min }^{M H}<c_{\min }$ and $c_{\max }^{M H}<c_{\max }$.

Proof. We have respectively

$$
\begin{aligned}
c_{\min }^{M H}-c_{\min } & =\left(1-p_{U}^{1}\right) v\left(b_{U}^{1}\right)+p_{U}^{1} v\left(d_{U}^{1}\right)-v\left(c_{U}^{0}\right)-\left(v\left(c_{U}^{1}\right)-v\left(c_{U}^{0}\right)\right) \\
& =\left(1-p_{U}^{1}\right) v\left(b_{U}^{1}\right)+p_{U}^{1} v\left(d_{U}^{1}\right)-v\left(c_{U}^{1}\right)<0
\end{aligned}
$$


and

$$
\begin{aligned}
c_{\max }^{M H}-c_{\max } & =\left(1-p_{H}^{1}\right) v\left(b_{H}^{1}\right)+p_{H}^{1} v\left(d_{H}^{1}\right)-v\left(c_{H}^{0}\right)-\left(v\left(c_{H}^{1}\right)-v\left(c_{H}^{0}\right)\right) \\
& =\left(1-p_{H}^{1}\right) v\left(b_{H}^{1}\right)+p_{H}^{1} v\left(d_{H}^{1}\right)-v\left(c_{H}^{1}\right)<0 .
\end{aligned}
$$

This result is due to the fact that as long as both contracts are actuarially fair, policyholders are always better off under the full insurance contract (Mossin, 1968). Roughly speaking, the partial coverage levels supplied by insurers to policyholders when they are unable to observe prevention effort reduce their expected utility when they do prevention. Therefore, the two threshold values are lower under the situation of unobserved prevention effort than under the assumption of perfect information.

The following result shows that both $c_{\min }^{M H}$ and $c_{\max }^{M H}$ are increasing in prevention efficiency.

\section{Result 12}

$$
\frac{\partial c_{\min }^{M H}}{\partial \Delta}>0 \text { and } \frac{\partial c_{\max }^{M H}}{\partial \Delta}>0
$$

Proof. See appendix.

The prevention efficiency $\Delta$ affects the threshold cost levels $c_{\min }^{M H}$ and $c_{\max }^{M H}$ through its impact on the utility level attained by agents who do make the prevention effort. This impact is twofold. The direct impact of a larger efficiency $\Delta$ lowers both the risk and premium, for a given coverage level $\alpha_{i}, i=\{U, H\}$ and thus increases the utility of this individual. The indirect impact of $\Delta$ takes place through variations in the coverage rate. If the coverage rate $\alpha_{i}$ increases with $\Delta$, this indirect impact reinforces the direct one. We show in the proof of Result 12 that, even if the coverage rate decreases with $\Delta$, the direct impact is larger than the indirect one, so that the value of the test always increases with $\Delta$ when $c_{\min }^{M H}<c<c_{\max }^{M H}$.

We now move to the value of the genetic test.

\subsection{To test or not to test}

The value of the test depends upon whether effort is undertaken at equilibrium -i.e., of how $c$ compares with $c_{\min }^{M H}$ and $c_{\max }^{M H}$. As in the previous section, we consider three cases according to the value taken by the prevention cost. 


\subsubsection{No one undertakes prevention: $c \geq c_{\max }^{M H}$}

In that case, an individual decides to take the test if

$$
\begin{aligned}
\Psi^{M H}(c, \Delta) & =\lambda U_{H}^{0}+(1-\lambda) U_{L}-U_{U}^{0}>0 \\
& \Leftrightarrow \lambda v\left(y-p_{H} d\right)+(1-\lambda) v\left(y-p_{L} d\right)-v\left(y-p_{U}^{0} d\right)>0
\end{aligned}
$$

which is never true, since $p_{U}^{0}$ is a linear combination of $p_{H}$ and $p_{L}$. Hence the following result.

Result 13 For $c \geq c_{\max }^{M H}, \Psi^{M H}(c, \Delta)<0, \forall(c, \Delta)$ so that the test is not taken.

This is the exact same result as in the preceding section (Result 4), which intuitive since we are back to the case where no prevention effort is undertaken, so that there is complete insurance at full coverage. We then have here that $\Psi^{M H}(c, \Delta)=\Psi_{0}<0$ for $c>c_{\max }^{M H}$, whatever the prevention efficiency level.

We now consider the case where even uninformed types make the prevention effort.

\subsubsection{Uninformed types undertake prevention: $c \leq c_{\min }^{M H}$}

Individuals decide to take the test if

$$
\begin{aligned}
\Psi^{M H}(c, \Delta) & =\lambda U_{H}^{1 M H}+(1-\lambda) U_{L}-U_{U}^{1 M H}>0 \\
& \Leftrightarrow \lambda\left[\left(1-p_{H}^{1}\right) v\left(b_{H}^{1}\right)+p_{H}^{1} v\left(d_{H}^{1}\right)-c\right]+(1-\lambda) v\left(c_{L}\right)-\left[\left(1-p_{U}^{1}\right) v\left(b_{U}^{1}\right)+p_{U}^{1} v\left(d_{U}^{1}\right)-c\right]>0
\end{aligned}
$$

We obtain the following Result.

Result 14 When $c \leq c_{\min }^{M H}$, we have that

$$
\begin{aligned}
& \text { (a) } \frac{\partial \Psi^{M H}(c, \Delta)}{\partial c}= 1-\lambda \\
&+\lambda\left[\left(1-p_{H}^{1}\right) p_{H}^{1} d \frac{\partial \alpha_{H}}{\partial c}\left[v^{\prime}\left(d_{H}^{1}\right)-v^{\prime}\left(b_{H}^{1}\right)\right]\right] \\
&-\left[p_{U}^{1}\left(1-p_{U}^{1}\right) d \frac{\partial \alpha_{U}}{\partial c}\left[v^{\prime}\left(d_{U}^{1}\right)-v^{\prime}\left(b_{U}^{1}\right)\right]\right] \\
& \lessgtr 1-\lambda=\frac{\partial \Psi(c, \Delta)}{\partial c}, \\
& \text { (b) } \frac{\partial \Psi^{M H}(c, \Delta)}{\partial c}>1-\lambda \text { if } \Delta \rightarrow \bar{\Delta},
\end{aligned}
$$




$$
\begin{aligned}
(c) \frac{\partial \Psi^{M H}}{\partial \Delta}= & \lambda d\left[\alpha_{H}\left(\left(1-p_{H}^{1}\right) v^{\prime}\left(b_{H}^{1}\right)+p_{H}^{1} v^{\prime}\left(d_{H}^{1}\right)\right)-\alpha_{U}\left(\left(1-p_{U}^{1}\right) v^{\prime}\left(b_{U}^{1}\right)+p_{U}^{1} v^{\prime}\left(d_{U}^{1}\right)\right)\right] \\
& +d\left[\lambda p_{H}^{1}\left(1-p_{H}^{1}\right) \frac{\partial \alpha_{H}}{\partial \Delta}\left(v^{\prime}\left(d_{H}^{1}\right)-v^{\prime}\left(b_{H}^{1}\right)\right)-p_{U}^{1}\left(1-p_{U}^{1}\right) \frac{\partial \alpha_{U}}{\partial \Delta}\left(v^{\prime}\left(d_{U}^{1}\right)-v^{\prime}\left(b_{U}^{1}\right)\right)\right] \\
& +\lambda\left[v\left(b_{H}^{1}\right)-v\left(d_{H}^{1}\right)-\left(v\left(b_{U}^{1}\right)-v\left(d_{U}^{1}\right)\right)\right] \\
\lessgtr & 0,
\end{aligned}
$$

d) $\Psi^{M H}(0, \Delta)=\Psi(0, \Delta)$ for all $\Delta$.

Proof. The proofs of parts a), c) and d) are straightforward, while the proof of b) is relegated to the appendix.

As in the situation without moral hazard, taking the test allows to save on the cost of effort in case the test is negative $-i$.e., with probability $1-\lambda$. As shown in part (a) above, moral hazard considerations introduce two additional terms, both related to how the coverage rate provided by insurance adapts to a larger $c$. Faced with a larger cost of effort, insurers have to decrease the coverage they propose to give an incentive for policy holders to exert the prevention effort. This in turn decreases both the utility of individuals who receive (with probability $\lambda$ ) a positive test and of those who do not take the test (and undertake prevention in all cases). The sign of the difference between these two affects is in general ambiguous, because types $U$ and $H$ differ both in coverage $\left(\alpha_{U}<\alpha_{H}\right)$ and in risk $\left(p_{H}^{1} \geq p_{U}^{1}\right)$. This makes it difficult to compare how the difference in marginal utilities between states of world (healthy or sick) is affected by the effort cost-induced variations of coverage. When $\Delta \rightarrow \bar{\Delta}$, the risks of both types converge when they undertake prevention, while the coverage rate remains lower for type $U$ than for type $H$ (because prevention is effective only with probability $\lambda$ for type $U$ ). We then obtain that a larger cost degrades more the utility of type $U$ than of type $H$, because there is a larger utility gap between states of the world for type $U$ (formally, $d_{U}^{1}<d_{H}^{1}<c_{U}^{1}=c_{H}^{1}<b_{H}^{1}<b_{U}^{1}$ ), who then suffers more at the margin from the decrease in coverage rate. This in turn increases the value of the test, compared to the case where prevention is observable.

As in the absence of moral hazard, a larger prevention effectiveness affects the utility of both types $H$ and $U$, but the effects are more convoluted with moral hazard. The first line of c) above corresponds to the same effect as without moral hazard, namely the difference in marginal (expected) utilities of both types, for given contracts. Nevertheless, this effect is more complex, because coverage is now incomplete for both types, and the sign of this term is ambiguous. The second line represents the impact of $\Delta$ on the difference in marginal utilities through endogenous variations in coverage rates for types $H$ and $U$ as $\Delta$ increases. Recall from Result 10 that this effect is ambiguous and depends mainly on the risk aversion of policyholders. Finally, the last line of c) 
represents the direct effect of $\Delta$ on the difference of utility levels of types $H$ and $U$, since a larger $\Delta$ makes the better state of the world (healthy outcome) more probable. We can show that, using incentive compatibility constraints (8) and (9), that this direct effect is negative. The sign of the sum of these three effects is ambiguous.

In a nutshell, the value of the test may not be increasing in prevention efficiency, because the utility of an uniformed type may increase more with $\Delta$ than that of a type $H$, due to the partial and endogenous coverage offered by insurers to both types in order to induce them to make the prevention effort. Finally, part d) is straightforward since the unobservability by insurers of the prevention effort does not matter when this effort is costless.

Observe that putting parts (b) and (d) of Result 14 together allows us to obtain the important following result.

Result 15 When $c \leq c_{\min }^{M H}$ and $\Delta \rightarrow \bar{\Delta}$, the value of the test is larger with than without moral hazard.

We will come back to (and extend) this result when assessing the impact of moral hazard on the value of the test in section 5 .

We then obtain the following result.

Result 16 When $c \leq c_{\min }^{M H}$, the value of the test is positive provided that the prevention efficiency $\Delta$ and the effort cost $c$ are large enough. Formally, assume that $\Delta$ is large enough. We then have that

a) there exists a (unique) value of $c$, denoted by $\tilde{c}_{1}^{M H}(\Delta)$, such that $\tilde{c}_{1}^{M H}(\Delta)<c_{\min }^{M H}$ and $\Psi^{M H}\left(\tilde{c}_{1}^{M H}(\Delta), \Delta\right)=0$. Moreover, $\tilde{c}_{1}^{M H}(\bar{\Delta})=0$;

b) $\Psi^{M H}(c, \Delta)<0$ for $c<\tilde{c}_{1}^{M H}(\Delta)$ and $\Psi^{M H}(c, \Delta)>0$ for $c>\tilde{c}_{1}^{M H}(\Delta)$.

This result is similar to the one obtained without moral hazard (Result 6) except that, since $\Psi^{M H}(c, \Delta)$ may not be increasing in $\Delta$ for all $\Delta$ and all $c<c_{m i n}^{M H}$, we are not able to provide a specific efficiency threshold above which there exist values of effort costs compatible with policyholders taking the test. We nevertheless know that this will be the case when the efficiency parameter $\Delta$ is close to its maximum $\bar{\Delta}$ (for the reasons explained after Result 14). We will compare the threshold costs in section 5 , and we now turn to the case where effort is undertaken if and only if the policyholder's type is high.

\subsubsection{Only informed types undertake prevention: $c_{\min }^{M H}<c<c_{\max }^{M H}$}

The value of the test for a policyholder is here given by

$$
\begin{aligned}
\Psi^{M H}(c, \Delta) & =\lambda U_{H}^{1 M H}+(1-\lambda) U_{L}-U_{U}^{0} \\
& =\lambda\left[\left(1-p_{H}^{1}\right) v\left(b_{H}^{1}\right)+p_{H}^{1} v\left(d_{H}^{1}\right)-c\right]+(1-\lambda) v\left(c_{L}\right)-v\left(c_{U}^{0}\right) .
\end{aligned}
$$


The next result states how prevention cost and efficiency affect the value of the test:

Result 17 For $c_{\min }^{M H}<c<c_{\max }^{M H}$, we have that

$$
\begin{aligned}
& \text { a) } \frac{\partial \Psi^{M H}(c, \Delta)}{\partial \Delta}>0, \\
& \text { b) } \frac{\partial \Psi^{M H}(c, \Delta)}{\partial c}<0 .
\end{aligned}
$$

Proof. Cf. appendix.

When $c_{\min }^{M H}<c<c_{\max }^{M H}$, the prevention efficiency $\Delta$ affects the value of the test only through its impact on the utility level attained by agents who obtain a positive test (and thus make the prevention effort). The impact on $\Psi^{M H}(c, \Delta)$ is then similar to the impact on $c_{\max }^{M H}$ (see Result 12 above). The impact of a higher prevention cost on the value of the test works similarly: the direct impact decreases the utility of the individual with a positive test (who then makes the prevention effort) for a given insurance contract, while the indirect impact of $c$ on the insurance contract is to decrease the coverage rate $\alpha_{H}$ proposed by the insurer (see Result 10), further damaging the utility of this individual and thus the value of the test.

Observe that the sign of the impact of $c$ and $\Delta$ on the value of the test is the same as without moral hazard: the moral hazard effects, through variations in the coverage rate $\alpha_{H}$, either reinforce the direct impact on the value of the test, in he case of $c$, or are swamped by the direct effect, in the case of $\Delta$. This is in stark contrast with the previous section, where the fact that moral hazard affects the insurance contracts offered to both types $H$ and $U$ (since they both undertake prevention and are offered insurance contracts with partial coverage) renders the sign of the impact of $c$ and $\Delta$ on the value of the test ambiguous.

We then obtain the following result.

Result 18 When $c_{\text {min }}^{M H} \leq c<c_{\text {max }}^{M H}$, the value of the test is positive provided that the prevention efficiency $\Delta$ is large while the effort cost $c$ is small. Formally, assume that $\Delta$ is large enough. We then have that

a) there exists a unique value of $c$, denoted by $\tilde{c}_{2}^{M H}(\Delta)$, such that $c_{\min }^{M H} \leq \tilde{c}_{2}^{M H}(\Delta)<c_{\max }^{M H}$ and $\Psi^{M H}\left(\tilde{c}_{2}^{M H}(\Delta), \Delta\right)=0$. Moreover, $c_{\min }^{M H}<\tilde{c}_{2}^{M H}(\bar{\Delta})<c_{\max }^{M H}$;

b) $\Psi^{M H}(c, \Delta)>0$ for $c_{\min }^{M H}<c<\tilde{c}_{2}^{M H}(\Delta)$;

c) $\tilde{c}_{2}^{M H}(\Delta)$ increases with $\Delta$.

This result is also similar to the one obtained without moral hazard (Result 8), with the same caveat as explained after Result 16, due to the ambiguity of the impact of prevention efficiency on the value of the test when only type $H$ makes the prevention effort. 
We now take stock of what we have learned when prevention is not observable, before moving to a direct comparison of our results with and without moral hazard in section 5 .

\subsection{Testing and effort at equilibrium}

We now summarize our results with unobservable prevention effort in the following propositions. They follow closely Propositions 1 and 2 obtained in the absence of moral hazard.

Proposition 3 A sufficient condition for the test to be taken is that the efficiency of prevention is large enough and that the prevention cost takes intermediate values: $\tilde{c}_{1}^{M H}(\Delta) \leq c \leq \tilde{c}_{2}^{M H}(\Delta)$. Moreover, the threshold $\tilde{c}_{2}^{M H}(\Delta)$ increases with $\Delta$.

The main difference with Proposition 1 is due to the fact that, as we have underlined in section 4.3.2, the value of the test need not always be increasing in the efficiency of prevention when the cost of prevention is low enough that even uninformed types take the test. This prevents us from determining a specific prevention efficiency threshold above which individuals take the test for specific values of prevention also. This also prevents us from assessing how the lowest prevention cost compatible with taking the test varies with the prevention efficiency. Except for these caveats, the main gist of our results is not affected by the introduction of moral hazard: the test is undertaken provided that the prevention efficiency is large enough, and that prevention costs take intermediate value. We defer to the next section for the comparison of the thresholds with and without moral hazard.

The following proposition states when preventions is undertaken as a function of its cost and efficiency and parallels Proposition 2.

Proposition 4 a) If the efficiency of prevention is large enough, then everyone undertakes prevention if its cost is low enough $\left(c<\tilde{c}_{1}^{M H}(\Delta)\right)$, only people of type $H$ undertake prevention if its cost is intermediate $\left(\tilde{c}_{1}^{M H}(\Delta) \leq c \leq \tilde{c}_{2}^{M H}(\Delta)\right)$ while no one makes a prevention effort otherwise (i.e., if $c>\tilde{c}_{2}^{M H}(\Delta)$ ).

b) If the efficiency of prevention is low enough that $\Psi^{M H}(c, \Delta)<0 \forall c$, then all agents undertake prevention if its cost is low enough $\left(c<c_{\text {min }}^{M H}\right)$ while no one undertakes prevention otherwise (if $c>c_{m i n}^{M H}$ ).

Similarly caveats apply for Proposition 4 as for Proposition 3, compared to the situation where prevention is observable. 
Figure 4 provides a graphical illustration of the value of the test as a function of prevention cost for four different values of the prevention efficiency. It is based on the same assumptions as those used to depict Figures 1 to 3, and is the equivalent, with moral hazard, to Figure 1.

\section{Insert Figure 4 around here}

Each curve on Figure 4 shows the value of the test as a function of prevention cost for a given value of prevention efficiency. All curves have the same shape, so we start by focusing on any curve $-i$.e., on any given efficiency $\Delta$. We observe that $\Psi^{M H}$ is first increasing and convex in $c$. This complements nicely our analytical finding (part b) of Result 14) that the slope of $\Psi^{M H}$ is larger than $1-\lambda$ when $\Delta \rightarrow \bar{\Delta}$. The curve $\Psi^{M H}$ is then (as proved in Result 17) decreasing in $c$ until it reaches $\Psi_{0}$ for $c>c_{\max }^{M H}$.

We now compare different curves -i.e., we study how the prevention efficiency $\Delta$ affects the value of the test. We have a numerical confirmation of the Result 14 that $\Psi^{M H}$ is indeed not increasing in $\Delta$ when $c<c_{m i n}^{M H}$. Figure 5 is indeed a subset of Figure 4 where we zoom on the low values of $c$. It shows that we have numerically a single crossing property, where a lower- $\Delta$ curve crosses a larger- $\Delta$ curve once and from below, meaning that $\Psi^{M H}$ increases with $\Delta$ for low values of $c$, and then decreases with $\Delta$.

Insert Figure 5 around here

A striking result from Figure 4 is that $\Psi^{M H}\left(c_{\text {min }}^{M H}, \Delta\right)$ is increasing in $\Delta$ for all $\Delta$ : although a larger prevention efficiency does not always increase the value of the test for all values of $c$ such that even untested types undertake effort, the maximum value of the test is indeed increasing with $\Delta$ in our numerical example. We also obtain numerically

(see Figure 5) that $\tilde{c}_{1}^{M H}$ decreases with $\Delta$ which, together with $\tilde{c}_{2}^{M H}(\Delta)$ increasing with $\Delta$ (see Proposition 3 above), means that the test is undertaken for a larger set of cost values as the efficiency of prevention increases. We had the same result without moral hazard, and we defer to next section the comparison of these thresholds with and without moral hazard.

\section{The impact of introducing moral hazard on testing and prevention}

We first assume that $\Delta$ is fixed, and look at how the testing and prevention decisions are affected by moral hazard as a function of the cost of prevention effort, $c$. We assume 
that $\Delta \rightarrow \bar{\Delta}$, and that $c_{\min }^{M H}<c_{\min }<c_{\max }^{M H}<c_{\max }$ (the case where $c_{\min }^{M H}<c_{\max }^{M H}<$ $c_{\min }<c_{\max }$ can be treated similarly and does not bring any new insight, so we leave it to the reader).

The following lemma will prove useful in what follows.

Lemma 3 We have (a) $\tilde{c}_{1}^{M H}(\Delta)<c<\tilde{c}_{1}(\Delta)$ and (b) $\tilde{c}_{2}^{M H}(\Delta)<c<\tilde{c}_{2}(\Delta)$ when $\Delta \rightarrow \bar{\Delta}$.

Roughly speaking, these results come from the fact that (a) the value of the test is larger with than without moral hazard when the effort cost is low enough that uninformed policyholders exert prevention effort while (b) it is lower when the effort cost is large enough that only type $H$ agents exert the effort. A consequence of Lemma 3 is that the interval of cost values that induce policyholders to exert effort in equilibrium is shifted to the left when moral hazard is introduced.

We then obtain the following proposition.

Proposition 5 Assume that $\Delta$ is large enough (close but not equal to $\bar{\Delta}$ ). Then (a) there exists a threshold $c_{\min }^{M H}<\hat{c}<c_{\min }$ such that the value of the test is larger (resp., lower) with than without moral hazard for all prevention costs below (resp., above) this threshold:

(b) for $\tilde{c}_{1}^{M H}(\Delta)<c<\min \left[\tilde{c}_{1}(\Delta), \tilde{c}_{2}^{M H}(\Delta)\right]$, the value of the test if positive with moral hazard but negative without: agents take the test if and only if there is moral hazard;

(c) for $\max \left[\tilde{c}_{1}(\Delta), \tilde{c}_{2}^{M H}(\Delta)\right]<c<\tilde{c}_{2}(\Delta)$, the value of the test if positive without moral hazard but negative with: agents take the test if and only if there is no moral hazard;

(d) the maximum value of the test is higher under moral hazard than without:

$$
\Psi^{M H}\left(c_{m i n}^{M H}, \Delta\right)>\Psi\left(c_{m i n}, \Delta\right) .
$$

We give the intuition for this important proposition, starting with part (a). Recall that the value of the test is defined as the difference between the expected utility of taking the test and of remaining uninformed about one's own risk. We know that the value of the test is larger with than without moral hazard when the effort cost is so low that even uninformed agents undertake the prevention effort (recall Result 15). The reason is that, moral hazard damages more the utility of the uninformed type than that of type $H$, through a lower coverage. ${ }^{14}$ On the opposite, the value of the test is lower with than without moral hazard when only type $H$ undertakes the prevention effort (i.e., for intermediate values of the prevention cost). In that case, uninformed agents receive the same contract (and thus utility level) with and without moral hazard. The contract

\footnotetext{
${ }^{14}$ As we explain after Result 14, this is true for $\Delta$ large enough that the main difference between these two types of agents is the coverage rate they buy, rather than their riskiness.
} 
offered to type $H$ with moral hazard is degraded compared to the situation without moral hazard because of the partial coverage offered, hence lowering the value of the test. And, of course, the value of the test is negative and minimum (corresponding to the utility loss from the discrimination risk) when the prevention cost is large enough that not even type $H$ undertake effort. Finally, since the value of the test is continuous in prevention cost whether prevention is observable or not, we can invoke the intermediate value theorem to obtain that there exists a cost threshold below (resp., above) which the value of the test is larger (resp., lower) with than without moral hazard.

Part (b) shows that, for some values of the prevention cost low enough that even uninformed agents undertake prevention, the value of the test is positive if and only if prevention is not observable. Recall that the value of the test is negative for very low values of the prevention cost (since the discrimination risk trumps any gain from acquiring information relevant to the cheap prevention effort), whether prevention is observable or not. The value of test is positive for larger amounts of the prevention cost, precisely because the information provided by the test becomes valuable in foregoing the costly prevention effort in the case the test is positive. In the case of moral hazard, taking the test also allows to receive a larger coverage in case one is revealed as type $H$. This reinforces the positive impact of a larger effort cost on the value of the test in case of moral hazard, so that it may be worth taking the test only in the case where prevention is not observable. Similarly, part (c) establishes that, for larger values of the effort cost (such that the value of the test is lower with than without moral hazard), agents undertake the test at equilibrium if and only if there is no moral hazard.

Finally, we give the intuition for part (d). In both cases (with and without moral hazard), the value of the test is measured for the prevention cost that renders uninformed agents indifferent between making the effort or not (anticipating the contract they obtain in each case). They also obtain the same contract in case of a negative test, or if they remain uninformed and do not exert any prevention. We then obtain that the value of the test can be expressed as a weighted average of, on the one hand, the utility difference between being of type $H$ or being uninformed, and making the prevention effort (weighted by the probability of being of type $H$ ) and, on the other hand, the utility difference between being of type $L$ and being uninformed, and not making the prevention effort (weighted by the probability of being of type $L$ ). Since the second part of this sum is not affected by moral hazard, the value of the test is larger with moral hazard if the difference in utility levels between uninformed and type $H$ agents is larger with moral hazard than without. We show that it is the case when prevention efficiency is close to its maximum, because, while the risks of the two types of agents converge in that case, the lower coverage offered by insurers to uninformed types (as opposed to type $H$ ) when prevention is not observable is especially detrimental to them.

With our numerical example, Proposition 5 holds for all values of $\Delta$, as illustrated in Figure 6 for the case where $\Delta=0.1<\bar{\Delta}=0.5$. 
Insert Figure 6 around here

We now endogenize the decision to take the test and study the impact of introducing moral hazard on the amount of prevention effort at equilibrium.

Proposition 6 Introducing moral hazard considerations (weakly) decreases the fraction of the population exerting the prevention effort.

To prove this proposition, observe first that, for values of $(c, \Delta)$ such that the testing decision is not affected by moral hazard, the fraction of the population exerting the prevention effort either remains constant or decreases. This is a straightforward consequence of the fact (see Result 11) that $c_{\min }^{M H}<c_{\min }$ and that $c_{\max }^{M H}<c_{\max }$. We now show that the same result holds if $(c, \Delta)$ is such that the introduction of moral hazard changes the testing decision. Proposition 5 has shown that two situations may occur. The first one happens when $(c, \Delta)$ are such that the test is taken if and only if there is moral hazard. This case materializes when the effort cost is low enough $\left(c<\tilde{c}_{1}(\Delta)<c_{\min }\right)$ that, without moral hazard, all individuals choose to remain uninformed and to undertake the prevention effort. The decision to take the test when moral hazard exists then induces low type agents not to exert the effort, decreasing the prevention effort (since $c<\tilde{c}_{2}^{M H}(\Delta)<c_{\max }^{M H}$ ). We then obtain that introducing moral hazard decreases the fraction of the population exerting the prevention effort at equilibrium by a factor $1-\lambda$. A similar phenomenon appears when the effort cost is large enough that agents take the test if and only if there is no moral hazard. The cost is large enough $\left(c>\tilde{c}_{2}^{M H}(\Delta)>c_{\min }^{M H}\right)$ that, with moral hazard, agents remain uninformed and do not exert effort while, without moral hazard, agents take the test and exert effort if they are of type $H$ (since $c<\tilde{c}_{2}(\Delta)<c_{\max }$ ). Hence, moral hazard also decreases prevention effort from a fraction $\lambda$ of the population to zero.

The analysis we have performed up to now in this section looks at the impact of introducing moral hazard for a given value of $\Delta$. We now look at how this impact varies as a function of $\Delta$. To complement our analytical results, we refer to Figure 7 (zoom on small values of $\Delta$ ). We now analytically that $\tilde{c}_{2}^{M H}(\Delta)<\tilde{c}_{2}(\Delta)$ and that $\tilde{c}_{1}^{M H}(\Delta)<\tilde{c}_{1}(\Delta)$ for $\Delta \rightarrow \bar{\Delta}$ (see Lemma 3 ). Figure 7 shows that these inequality hold for all values of $\Delta$ with our numerical simulation. ${ }^{15}$ We also know that $\tilde{c}_{1}(\Delta)$ decreases with $\Delta$ (Result 6 ), while $\tilde{c}_{2}^{M H}(\Delta)$ and $\tilde{c}_{2}(\Delta)$ increase with $\Delta$ for any $\Delta$ (respectively, Results 18 and $8(\mathrm{c}))$. Figure 7 shows that $\tilde{c}_{1}^{M H}(\Delta)$ also increases with $\Delta$ within our numerical example.

\footnotetext{
${ }^{15} \tilde{c}_{1}$ and $\tilde{c}_{1}^{M H}$ are depicted in blue in Figure 7 , while $\tilde{c}_{2}$ and $\tilde{c}_{2}^{M H}$ are depicted in purple.
} 
Insert Figure 7

Finally, Figure 7 shows that the minimum value of $\Delta$ above which there exists an interval of prevention cost values compatible with taking the test is lower (at 3.4\%) with than without moral hazard (where $\tilde{\Delta}=4 \%$ ). We summarize this result in the next proposition:

Proposition 7 Introducing moral hazard considerations may induce individuals to undertake the genetic test for lower values of the prevention efficiency $\Delta$.

The intuition for this proposition is that the value of the test is larger with than without moral hazard when the prevention cost is low enough that uninformed agents undertake the effort. As explained above, the test value is larger because moral hazard degrades especially the contract (and utility) that is offered to uninformed agents in order to induce them to make the (unobservable) effort. We have shown analytically (see Result 14) that this is true when $\Delta \rightarrow \bar{\Delta}-i . e .$, when the difference in coverage between uninformed and type $H$ policyholders is larger than the difference in risk between them. The numerical results on which the figures reported here are drawn show that it is still the case when the efficiency of prevention effort is low.

Up to now, we have concentrated on the value of the test, and on the testing and prevention decisions of agents. We now look at their welfare level.

\section{Welfare analysis}

In this section, we investigate the impact of the availability of (observable or not) prevention effort, testing and insurance on the ex ante welfare of agents. We start from the simplest case where prevention is not available, and we then sequentially the availability of prevention, of testing and of risk discrimination insurance in order to measure their individual impact on welfare. We illustrate our results with the help of Figures 8 and 9 , which depict welfare (ex ante utility) as a function of the prevention $\operatorname{cost} c$, for a given value of $\Delta$, under various scenarios.

\section{Insert Figure 8}

We start from the simplest situation where prevention is not available. In that case, policyholders do not take the test since the test has only drawbacks, namely the discrimination risk. The ex ante utility level is then $v\left(c_{U}^{0}\right)$ which is of course independent 
of $c$. This utility level corresponds to the horizontal line on Figure $8 .{ }^{16}$ We then introduce the possibility to exert effort but assume that the genetic test is not available. In that case, agents are uninformed about their individual risk and exert effort if and only if the effort cost is lower than the threshold $c_{\min }$ (see Result 2). In case of effort, their ex ante utility is given by $v\left(c_{U}^{1}\right)-c$ for $c<c_{\max }$, and $v\left(c_{U}^{0}\right)$ for $c \geq c_{\max }$. We represent this utility level on Figure 8. The vertical distance between this utility level and the horizontal line (denoted by A on Figure 8) represents the ex ante utility gain from the prevention technology. It obviously decreases linearly (at a rate of one) with the cost of effort.

The next step consists in introducing the testing technology, assuming that the prevention effort is observable and the prevention efficiency $\Delta$ is large enough that the test is worth taking for certain values of $c$. We know from Result 3 that the test is taken only if the effort cost $c$ is comprised between $\tilde{c}_{1}$ and $\tilde{c}_{2}$. For $c<\tilde{c}_{1}$, agents remain uninformed and exert effort, so that their utility remains $v\left(c_{U}^{1}\right)-c$, while if $c>\tilde{c}_{2}$ they also remain uninformed but do not exert effort, with a utility level of $v\left(c_{U}^{0}\right)$. For $c$ in between $\tilde{c}_{1}$ and $\tilde{c}_{2}$, their ex ante utility is $\lambda\left(v\left(c_{H}^{1}\right)-c\right)+(1-\lambda) v\left(c_{L}\right)$, which decreases with $c$ at a a rate of $1-\lambda$ since the test allows to forego the cost of effort when the test is negative. Figure 8 depicts the value of the test as a function of the cost of prevention (vertical distance labeled B). It is composed of the gain from the targeted effort, minus the discrimination risk.

Before turning to the impact of moral hazard, we show on Figure 8 the loss of ex ante welfare associated with the discrimination risk entailed by taking the test in our setting. If there were no discrimination risk, agents would undertake the test in order to exert the prevention effort only when they are revealed to be of a high type. It is easy to see that their ex ante utility level in case where the prevention cost is zero is not affected by whether they take the test or not. This utility level then decreases at a rate of $1-\lambda$ with the effort cost, since the test allows to forego this cost when the test is negative. For very large values of $c$, agents prefer not to make the effort and then obtain the utility level $v\left(c_{U}^{0}\right)$. We then represent on Figure 8 the ex ante utility cost associated with the discrimination risk embedded into the test in our setting (vertical distance C). Not surprisingly, this discrimination risk (i) means that the test is taken for a smaller interval of prevention cost and (ii) decreases the value of the test even for the interval of cost where the test is taken when the discrimination risk exists.

We now turn to Figure 9, which depicts the impact of making the prevention effort unobservable when the testing technology is available (but entails a discrimination risk). We know from Lemma 3 that moral hazard reduces the two cost thresholds below which, respectively, policyholders exert effort even if uninformed about their own risk level

\footnotetext{
${ }^{16}$ This level is larger than the expected $e x$ ante utility in case no insurance exists, which is given by $p_{U}^{0} v(y-d)+\left(1-p_{U}^{0}\right) v(y)$.
} 
$\left(c_{\min }^{M H}<c_{\min }\right)$ and if they know their type to be $H\left(c_{\max }^{M H}<c_{\max }\right)$. Moreover, the $e x$ ante utility is lower with moral hazard, even when the testing decision is the same than without moral hazard, because of the lower coverage implied by the unobservability of the prevention effort. Figure 9 represents this welfare loss of moral hazard as the vertical distance $\mathrm{D}$ between the two curves.

Insert Figure 9

\section{Conclusion}

We have studied the situation where a free genetic test perfectly informs an individual about his risk of developing a specific disease in the future. This information allows the individual to undertake a costly prevention effort, which reduces his probability of incurring the health damage in the case the genetic test is positive. The drawback of the genetic test is that its results are used by insurers to price their insurance policies, so that agents undertaking the test are faced with a discrimination risk. We first show that, when the prevention effort is observable, the pros of the test are larger than its cons when the prevention cost is neither too low nor too high. We then obtain that, when effort is not observable by insurers, the private value of the genetic test is not always increasing with the efficiency of prevention. Also, and contrary to the intuition, the value of the test may actually be larger when effort is not observable, so that the test may be taken for lower values of the prevention efficiency than when prevention is observable.

Our analysis could be extended in several directions. In line with Barrigozzi and Henriet (2011), it would be interesting to compare different regulatory approaches in order to highlight in a same framework the interplay between risk discrimination, moral hazard and adverse selection. Also, we could assume that individuals differ in their "repulsion to chance" (to borrow Hoel et al. (2006)'s terminology), so that some may undertake the test even if its information value if negative, while others would not even if the value is positive. 


\section{References}

[1] Bardey D. and R. Lesur, 2005, "Optimal health insurance contract: Is a deductible useful?," Economics Letters, vol. 87(3), p. 313-317.

[2] Barigozzi F. and D. Henriet [2011], "Genetic information: comparing alternative regulatory approaches when prevention matters", Journal of Public Economic Theory, 13(1), p. 23-46.

[3] Collins F., 2010, The Language of Life: DNA and the Revolution in Personalized Medicine, kindle edition, HarperCollins Publishers.

[4] Crainich D., 2011, "Self-insurance with genetic testing tools", Working paper Lem.

[5] Davies K., 2010, The $\$ 1,000$ genome, Free Press.

[6] Doherty N. and L. Posey 1998, "On the value of a checkup: adverse selection, moral hazard and the value of information", The Journal of Risk and Insurance, 65(2), p. 189-211.

[7] Doherty N. and P. Thistle 1996, "Adverse selection with endogenous information in insurance market", Journal of Public Economics, 63, p. 83-102.

[8] Ehrlich I. and G. Becker 1972, "Market insurance, self-insurance and selfprotection", Journal of Political Economy, 80, p. 623-648.

[9] Hirshleifer J. 1971, "The Private and Social Value of Information and the Reward to Incentive Activity", The American Economic Review, 61, p. 561-74.

[10] Filipova L and M. Hoy, 2009, "Impact of genetic testing on surveillance and prevention", Working paper University of Guelph.

[11] Hoel M. and T. Iversen 2002, "Genetic testing when there is a mix of compulsory and voluntary health insurance" Journal of Health Economics, 21(2), p. 253-270.

[12] Hoel M. and Iversen T, Nilssen T. and Vislie J., 2006, "Genetic testing in competitive insurance markets with repulsion from chance: A welfare analysis", Journal of Health Economics, 25, p. 847-860.

[13] Hoy M. 1989, "The value of screening mecanisms under alternative insurance possibilities", Journal of Public Economics, 39, p. 177-206.

[14] Hoy M. and Polborn M., 2000, "The value of genetic information in the life insurance market", Journal of Public Economics, 78, p. 235-252. 
[15] Mossin J., 1968, "Aspects of rational insurance purchasing," Journal of Political Economy, 76, p. 533-568.

[16] Rees R. and P. Apps, 2006, "Genetic testing, income distribution and insurance markets", Les Annales d'Economie et de Statistique, 83-84, p. 295-325.

[17] Shavell S., 1979, "On moral hazard and insurance," Quarterly Journal of Economics, vol 93, No 4, p. 541-562.

[18] Strohmenger R. and A. Wambach 2000, "Adverse selection and categorical discrimination in the health insurance markets: the effects of genetic tests", Journal of Health Economics, 19(2), p. 197-218.

[19] Tabarrok, A. 1994, "Genetic testing: an economic and contractarian analysis", Journal of Health Economics, 13, p. 75-91.

\section{Appendix}

\subsection{Proof of Result 6}

Proof. a) First, we know from Result 1 that $c_{\min }=0$ if $\Delta=0$ and from Result 5 that $\Psi(0,0)<0$. Second, we also know that $\partial c_{\min } / \partial \Delta>0$ (so that $c_{\min }>0$ if $\Delta>0$ ) which, together with $\partial \Psi(c, \Delta) / \partial c>0$ and $\partial \Psi(c, \Delta) / \partial \Delta>0$, implies that

$$
\frac{d \Psi\left(c_{\text {min }}, \Delta\right)}{d \Delta}=\frac{\partial \Psi\left(c_{\min }, \Delta\right)}{\partial c} \frac{\partial c_{\text {min }}}{\partial \Delta}+\frac{\partial \Psi\left(c_{\text {min }}, \Delta\right)}{\partial \Delta}>0 .
$$

Finally, we know that $\Psi(0, \bar{\Delta})=0$ and that $c_{\min }>0$ when $\Delta=\bar{\Delta}$, which imply that $\Psi\left(c_{\min }, \bar{\Delta}\right)>0$. The continuity of $\Psi(c, \Delta)$ in $\Delta$ together with the fact that $\Psi(c, \Delta)$ is strictly increasing with $\Delta$ for any $c$ implies, by the intermediate value theorem, that there exists a unique value $0<\Delta<\bar{\Delta}$, denoted by $\tilde{\Delta}$, such that $\Psi\left(c_{\min }, \tilde{\Delta}\right)=0$.

b) By the same reasoning as above, we know that $\Psi\left(c_{m i n}, \Delta\right)>0$ for all $\Delta>\tilde{\Delta}$. The fact that $\partial \Psi(c, \Delta) / \partial c>0$ and that $\Psi(0, \Delta) \leq 0$ for all $\Delta>\tilde{\Delta}$ imply, by the intermediate value theorem, that there exists a unique value of $c$, denoted by $\tilde{c}_{1}(\Delta)$, such that $0 \leq \tilde{c}_{1}(\Delta) \leq c_{\min }$ and $\Psi\left(\tilde{c}_{1}(\Delta), \Delta\right)=0$;

c) Straightforward since $\partial \Psi(c, \Delta) / \partial c>0$.

d) We have by definition that $\Psi\left(\tilde{c}_{1}(\Delta), \Delta\right)=0$ so that

$$
\frac{d \Psi\left(\tilde{c}_{1}(\Delta), \Delta\right)}{d \Delta}=\frac{\partial \Psi\left(\tilde{c}_{1}(\Delta), \Delta\right)}{\partial \tilde{c}_{1}(\Delta)} \frac{\partial \tilde{c}_{1}(\Delta)}{\partial \Delta}+\frac{\partial \Psi\left(\tilde{c}_{1}(\Delta), \Delta\right)}{\partial \Delta}=0 .
$$

Our claim then results from the fact that $\partial \Psi(c, \Delta) / \partial c>0$ and that $\partial \Psi(c, \Delta) / \partial \Delta>0$ for all $c$ and $\Delta$.

e) Straightforward since part b) of Result 5 shows that $\Psi(0, \bar{\Delta})=0$. 


\subsection{Proof of Result 8}

\section{Proof.}

a) First, part a) of the proof of Result 6 has shown that $\Psi\left(c_{m i n}, \Delta\right)>0$ for all $\Delta>\tilde{\Delta}$. Second, Result 4 has shown that $\Psi\left(c_{\max }, \Delta\right)<0$ for all $\Delta$. The fact that $\partial \Psi(c, \Delta) / \partial c<$ 0 then implies, by the intermediate value theorem, that there exists a unique value of $c$, denoted by $\tilde{c}_{2}(\Delta)$, such that $c_{\min } \leq \tilde{c}_{2}(\Delta)<c_{\max }$ and $\Psi\left(\tilde{c}_{2}(\Delta), \Delta\right)=0$;

b) Straightforward since $\partial \Psi(c, \Delta) / \partial c<0$.

c) We have by definition that $\Psi\left(\tilde{c}_{2}(\Delta), \Delta\right)=0$ so that

$$
\frac{d \Psi\left(\tilde{c}_{2}(\Delta), \Delta\right)}{d \Delta}=\frac{\partial \Psi\left(\tilde{c}_{2}(\Delta), \Delta\right)}{\partial c} \frac{\partial \tilde{c}_{2}(\Delta)}{\partial \Delta}+\frac{\partial \Psi\left(\tilde{c}_{2}(\Delta), \Delta\right)}{\partial \Delta}=0 .
$$

Our claim then results from the fact that $\partial \Psi(c, \Delta) / \partial c<0$ and that $\partial \Psi(c, \Delta) / \partial \Delta>0$ for all $c$ and $\Delta$.

d) The fact that $\tilde{c}_{1}(\tilde{\Delta})=\tilde{c}_{2}(\tilde{\Delta})=c_{m i n}$ comes from the definitions of $\tilde{\Delta}, \tilde{c}_{1}(\Delta)$ and $\tilde{c}_{2}(\Delta)$. The fact that $c_{\min }<\tilde{c}_{1}(\bar{\Delta})<c_{\max }$ comes from the observation that $\Psi\left(c_{\min }, \bar{\Delta}\right)>0$ while $\Psi\left(c_{\max }, \bar{\Delta}\right)<0$.

\subsection{Proof of Result 9}

Proof. $\alpha_{H}$ and $\alpha_{U}$ are respectively implicitly determined by

$$
c=\left(p_{H}^{0}-p_{H}^{1}\right)\left(v\left(b_{H}^{1}\right)-v\left(d_{H}^{1}\right)\right)
$$

and,

$$
c=\lambda\left(p_{H}^{0}-p_{H}^{1}\right)\left(v\left(b_{U}^{1}\right)-v\left(d_{U}^{1}\right)\right) .
$$

It is worth noticing that $\alpha_{U}=\alpha_{H}$ in the special case $\lambda=1\left(\right.$ since $\left.p_{U}^{1}=p_{H}^{1}\right)$. Then, let us consider the following function

$$
F\left(\alpha_{U}, \lambda\right)=\lambda\left(p_{H}^{0}-p_{H}^{1}\right)\left(v\left(b_{U}^{1}\right)-v\left(d_{U}^{1}\right)\right)-c .
$$

The implicit function theorem gives

$$
\begin{aligned}
\frac{d \alpha_{U}}{d \lambda} & =-\frac{\partial F\left(\alpha_{U}, \lambda\right) / \partial \lambda}{\partial F\left(\alpha_{U}, \lambda\right) / \partial \alpha_{U}} \\
& =\frac{\left(p_{H}^{0}-p_{H}^{1}\right)\left[v\left(b_{U}^{1}\right)-v\left(d_{U}^{1}\right)+\lambda \alpha_{U}\left(p_{H}^{1}-p_{L}\right) d\left(v^{\prime}\left(d_{U}^{1}\right)-v^{\prime}\left(b_{U}^{1}\right)\right)\right]}{\lambda \Delta\left(p_{U}^{1} v^{\prime}\left(b_{U}^{1}\right)+\left(1-p_{U}^{1}\right) v^{\prime}\left(d_{U}^{1}\right)\right)}>0 .
\end{aligned}
$$




\subsection{Proof of Result 10}

Proof. The level of coverage $\alpha_{H}$ is determined by

$$
c=\left(p_{H}^{0}-p_{H}^{1}\right)\left(v\left(b_{H}^{1}\right)-v\left(d_{H}^{1}\right)\right) .
$$

a) The implicit function theorem implies:

$$
\frac{\partial \alpha_{H}}{\partial c}=-\frac{1}{\Delta d\left[p_{H}^{1} v^{\prime}\left(b_{H}^{1}\right)+\left(1-p_{H}^{1}\right) v^{\prime}\left(d_{H}^{1}\right)\right]}<0 .
$$

The coverage rate $\alpha_{H}$ attains the minimum value of zero when

$$
c=\bar{c}_{H}=\Delta(v(y)-v(y-d)) .
$$

We proceed similarly to prove that $\alpha_{U}$ is decreasing in $c$, and that the minimum value of $\alpha_{U}=0$ is reached when

$$
c=\bar{c}_{U}=\lambda \Delta(v(y)-v(y-d))
$$

so that $\bar{c}_{U}<\bar{c}_{H}$.

b) The implicit function theorem implies:

$$
\frac{\partial \alpha_{H}}{\partial \Delta}=\frac{v\left(b_{H}^{1}\right)-v\left(d_{H}^{1}\right)+\Delta \alpha_{H} d\left[v^{\prime}\left(b_{H}^{1}\right)-v^{\prime}\left(d_{H}^{1}\right)\right]}{\Delta d\left[p_{H}^{1} v^{\prime}\left(b_{H}^{1}\right)+\left(1-p_{H}^{1}\right) v^{\prime}\left(d_{H}^{1}\right)\right]} .
$$

The denominator is always positive. The numerator is positive if and only if

$$
-\frac{v\left(b_{H}^{1}\right)-v\left(d_{H}^{1}\right)}{\left[v^{\prime}\left(b_{H}^{1}\right)-v^{\prime}\left(d_{H}^{1}\right)\right]} \frac{1}{\Delta d} \geq \alpha_{H} .
$$

The generalized mean value theorem implies

$$
-\frac{v^{\prime}\left(\chi_{H}\right)}{v^{\prime \prime}\left(\chi_{H}\right)} \frac{1}{\Delta d} \geq \alpha_{H}
$$

with $\chi_{H} \in\left[d_{H}^{1}, b_{H}^{1}\right]$. The same reasoning applies for $\alpha_{U}$.

\subsection{Proof of Result 12}

Proof. a) The derivative of $c_{\max }^{M H}$ with respect to $\Delta$ is

$$
\begin{aligned}
\frac{\partial c_{\max }^{M H}}{\partial \Delta}= & v\left(b_{H}^{1}\right)-v\left(d_{H}^{1}\right)+\alpha_{H} d\left[\left(1-p_{H}^{1}\right) v^{\prime}\left(b_{H}^{1}\right)+p_{H}^{1} v^{\prime}\left(d_{H}^{1}\right)\right] \\
& +d \frac{\partial \alpha_{H}}{\partial \Delta}\left(1-p_{H}^{1}\right) p_{H}^{1}\left(v^{\prime}\left(d_{H}^{1}\right)-v^{\prime}\left(b_{H}^{1}\right)\right)
\end{aligned}
$$


If $\partial \alpha_{H} / \partial \Delta>0$, it is clear that $\partial c_{\max }^{M H} / \partial \Delta>0$. On the contrary, if $\partial \alpha_{H} / \partial \Delta<0$, the sign is a priori ambiguous. We have

$$
\begin{aligned}
\frac{\partial c_{\max }^{M H}}{\partial \Delta}= & v\left(b_{H}^{1}\right)-v\left(d_{H}^{1}\right)+\alpha_{H} d v^{\prime}\left(b_{H}^{1}\right) \\
& +\alpha_{H} d p_{H}^{1}\left[v^{\prime}\left(d_{H}^{1}\right)-v^{\prime}\left(b_{H}^{1}\right)\right]+d \frac{\partial \alpha_{H}}{\partial \Delta}\left(1-p_{H}^{1}\right) p_{H}^{1}\left(v^{\prime}\left(d_{H}^{1}\right)-v^{\prime}\left(b_{H}^{1}\right)\right) .
\end{aligned}
$$

A sufficient condition to have $\partial c_{\max }^{M H} / \partial \Delta>0$ is then

$$
\alpha_{H}+\frac{\partial \alpha_{H}}{\partial \Delta}\left(1-p_{H}^{1}\right) \geq 0
$$

According to Result 10, we have

$$
\frac{d \alpha_{H}}{d \Delta}=\frac{v\left(b_{H}^{1}\right)-v\left(d_{H}^{1}\right)+\Delta \alpha_{H} d\left[v^{\prime}\left(b_{H}^{1}\right)-v^{\prime}\left(d_{H}^{1}\right)\right]}{\Delta d\left[p_{H}^{1} v^{\prime}\left(b_{H}^{1}\right)+\left(1-p_{H}^{1}\right) v^{\prime}\left(d_{H}^{1}\right)\right]},
$$

whose denominator is always positive. Therefore, the previous sufficient condition becomes

$$
\begin{aligned}
& \alpha_{H} \geq-\left(\frac{v\left(b_{H}^{1}\right)-v\left(d_{H}^{1}\right)+\Delta \alpha_{H} d\left[v^{\prime}\left(b_{H}^{1}\right)-v^{\prime}\left(d_{H}^{1}\right)\right]}{\Delta d\left[p_{H}^{1} v^{\prime}\left(b_{H}^{1}\right)+\left(1-p_{H}^{1}\right) v^{\prime}\left(d_{H}^{1}\right)\right]}\right)\left(1-p_{H}^{1}\right) \\
\Leftrightarrow & \Delta d \alpha_{H}\left[p_{H}^{1} v^{\prime}\left(b_{H}^{1}\right)+\left(1-p_{H}^{1}\right) v^{\prime}\left(d_{H}^{1}\right)+\left[v^{\prime}\left(b_{H}^{1}\right)-v^{\prime}\left(d_{H}^{1}\right)\right]\left(1-p_{H}^{1}\right)\right] \geq-\left(v\left(b_{H}^{1}\right)-v\left(d_{H}^{1}\right)\right)\left(1-p_{H}^{1}\right) \\
\Leftrightarrow & \Delta d \alpha_{H} v^{\prime}\left(b_{H}^{1}\right) \geq-\left(v\left(b_{H}^{1}\right)-v\left(d_{H}^{1}\right)\right)\left(1-p_{H}^{1}\right),
\end{aligned}
$$

which is always true since the RHS is negative.

We can proceed similarly to prove that $\partial c_{m i n}^{M H} / \partial \Delta>0$.

\subsection{Proof of part b) of Result 14}

Proof. We have that

$$
\begin{aligned}
& \frac{\partial \Psi^{M H}(c, \Delta)}{\partial c} \geq \frac{\partial \Psi(c, \Delta)}{\partial c}=1-\lambda \\
& \Longleftrightarrow \lambda\left[\frac{\left(1-p_{H}^{1}\right) p_{H}^{1}\left[v^{\prime}\left(d_{H}^{1}\right)-v^{\prime}\left(b_{H}^{1}\right)\right]}{\left[p_{H}^{1} v^{\prime}\left(b_{H}^{1}\right)+\left(1-p_{H}^{1}\right) v^{\prime}\left(d_{H}^{1}\right)\right]}\right] \leq\left[\frac{p_{U}^{1}\left(1-p_{U}^{1}\right)\left[v^{\prime}\left(d_{U}^{1}\right)-v^{\prime}\left(b_{U}^{1}\right)\right]}{\lambda\left[p_{U}^{1} v^{\prime}\left(b_{U}^{1}\right)+\left(1-p_{U}^{1}\right) v^{\prime}\left(d_{U}^{1}\right)\right]}\right] .
\end{aligned}
$$

Let us consider the case where $\Delta \rightarrow \bar{\Delta}$.In such a case, we obtain

$$
\begin{aligned}
& \frac{\partial \Psi^{M H}(c, \Delta)}{\partial c} \geq \frac{\partial \Psi(c, \Delta)}{\partial c} \\
\Longleftrightarrow & \lambda^{2}\left[\frac{v^{\prime}\left(d_{H}^{1}\right)-v^{\prime}\left(b_{H}^{1}\right)}{p_{L} v^{\prime}\left(b_{H}^{1}\right)+\left(1-p_{L}\right) v^{\prime}\left(d_{H}^{1}\right)}\right] \leq\left[\frac{v^{\prime}\left(d_{U}^{1}\right)-v^{\prime}\left(b_{U}^{1}\right)}{p_{L} v^{\prime}\left(b_{U}^{1}\right)+\left(1-p_{L}\right) v^{\prime}\left(d_{U}^{1}\right)}\right] .
\end{aligned}
$$


A sufficient condition is

$$
\begin{aligned}
& {\left[p_{L} v^{\prime}\left(b_{U}^{1}\right)+\left(1-p_{L}\right) v^{\prime}\left(d_{U}^{1}\right)\right]\left[v^{\prime}\left(d_{H}^{1}\right)-v^{\prime}\left(b_{H}^{1}\right)\right] \leq\left[p_{L} v^{\prime}\left(b_{H}^{1}\right)+\left(1-p_{L}\right) v^{\prime}\left(d_{H}^{1}\right)\right]\left[v^{\prime}\left(d_{U}^{1}\right)-v^{\prime}\left(b_{U}^{1}\right)\right], } \\
\Longleftrightarrow & v^{\prime}\left(d_{U}^{1}\right) v^{\prime}\left(b_{H}^{1}\right) \geq v^{\prime}\left(b_{U}^{1}\right) v^{\prime}\left(d_{H}^{1}\right),
\end{aligned}
$$

which is true since $d_{U}^{1}<d_{H}^{1}$ for $\Delta \rightarrow \bar{\Delta}$.

\subsection{Proof of Result 16}

Proof. a) Start by assuming that $\Delta=\bar{\Delta}$. We know from Result $14 \mathrm{~b}$ ) that

$$
\Psi^{M H}(0, \bar{\Delta})=\Psi(0, \bar{\Delta})=0 .
$$

Part b) of Result 14 shows that

$$
\frac{\partial \Psi^{M H}(c, \bar{\Delta})}{\partial c} \geq \frac{\Psi(c, \bar{\Delta})}{\partial c}=1-\lambda \text { for all } c<c_{m i n}^{M H},
$$

which implies that

$$
\Psi^{M H}(c, \bar{\Delta})>0 \text { for all } c<c_{m i n}^{M H} .
$$

We then have that $\tilde{c}_{1}^{M H}(\bar{\Delta})=0$.

Assume now that $\Delta<\bar{\Delta}$ while remaining close enough. Observe that, by continuity of $\Psi$ and $\Psi^{M H}$ in $c$, we have that

$$
\frac{\partial \Psi^{M H}(c, \Delta)}{\partial c} \geq \frac{\Psi(c, \Delta)}{\partial c}=1-\lambda \text { for all } c<c_{\text {min }}^{M H} \text { and } \Delta \rightarrow \bar{\Delta} .
$$

We know from Result $14 \mathrm{~b}$ ) that

$$
\Psi^{M H}(0, \Delta)=\Psi(0, \Delta)<0 .
$$

Since $\Psi^{M H}(c, \Delta)$ is continuous in $c$, we have that

$$
\Psi^{M H}\left(c_{m i n}^{M H}, \Delta\right)>0
$$

By the intermediate value theorem, there exists a unique value of $c$, denoted by $\tilde{c}_{1}^{M H}(\Delta)$, such that $\tilde{c}_{1}^{M H}(\Delta)<c_{m i n}^{M H}$ and $\Psi^{M H}\left(\tilde{c}_{1}^{M H}(\Delta), \Delta\right)=0$.

b) The proof is straightforward by definition of $\tilde{c}_{1}^{M H}(\Delta)$ and by the intermediate value theorem. 


\subsection{Proof of Result 17}

Proof.

a) Observe that, for $c_{\min }^{M H}<c<c_{\max }^{M H}$, we have

$$
\Psi^{M H}(c, \Delta)=\lambda\left(c_{\max }^{M H}-c+v\left(c_{H}^{0}\right)\right)+(1-\lambda) v\left(c_{L}\right)-v\left(c_{U}^{0}\right),
$$

so that

$$
\frac{\partial \Psi^{M H}(c, \Delta)}{\partial \Delta}=\lambda \frac{\partial c_{\max }^{M H}}{\partial \Delta}>0
$$

by Result 12 .

b) The derivative of $\Psi^{M H}(c, \Delta)$ with respect to $c$ is

$$
\begin{aligned}
\frac{\partial \Psi^{M H}(c, \Delta)}{\partial c} & =\lambda\left(-1-\left(1-p_{H}^{1}\right) v^{\prime}\left(b_{H}^{1}\right) \frac{\partial \alpha_{H}}{\partial c} p_{H}^{1} d+p_{H}^{1} v^{\prime}\left(d_{H}^{1}\right) \frac{\partial \alpha_{H}}{\partial c}\left(1-p_{H}^{1}\right) d\right) \\
& =\lambda\left(p_{H}^{1}\left(1-p_{H}^{1}\right) d \frac{\partial \alpha_{H}}{\partial c}\left[v^{\prime}\left(d_{H}^{1}\right)-v^{\prime}\left(b_{H}^{1}\right)\right]-1\right)<0 .
\end{aligned}
$$

\subsection{Proof of Result 18}

Proof. a) Recall that, for $c_{\min }^{M H}<c<c_{\max }^{M H}$, we have

$$
\Psi^{M H}(c, \bar{\Delta})=(1-\lambda) v\left(c_{L}\right)+\lambda\left[\left(1-p_{L}\right) v\left(b_{H}^{1}\right)+p_{L} v\left(d_{H}^{1}\right)-c\right]-v\left(c_{U}^{0}\right) .
$$

Result 13 has shown that $\Psi^{M H}\left(c_{\max }^{M H}, \bar{\Delta}\right)<0$. We now prove that $\Psi^{M H}\left(c_{\min }^{M H}, \bar{\Delta}\right)>0$. We have

$$
\begin{aligned}
\Psi^{M H}\left(c_{m i n}^{M H}, \bar{\Delta}\right)= & (1-\lambda)\left[v\left(c_{L}\right)-v\left(c_{U}^{0}\right)\right] \\
& +\lambda\left[p_{L}\left[v\left(d_{H}^{1}\right)-v\left(d_{U}^{1}\right)\right]+\left(1-p_{L}\right)\left[v\left(b_{H}^{1}\right)-v\left(b_{U}^{1}\right)\right]\right] .
\end{aligned}
$$

Note that the first term of (12) is positive. Moreover, using the mean value theorem, we obtain that

$$
\begin{aligned}
& v\left(d_{H}^{1}\right)-v\left(d_{U}^{1}\right)=v^{\prime}(\varpi) d\left(1-p_{L}\right)\left(\alpha_{H}-\alpha_{U}\right), \\
& v\left(b_{H}^{1}\right)-v\left(b_{U}^{1}\right)=v^{\prime}(\nu) d p_{L}\left(\alpha_{U}-\alpha_{H}\right),
\end{aligned}
$$

with $\varpi \in\left[d_{H}^{1}, d_{U}^{1}\right]$ and $\nu \in\left[b_{H}^{1}, b_{U}^{1}\right]$. Therefore, the second term of (12) becomes

$$
\begin{aligned}
& p_{L}\left[v\left(d_{H}^{1}\right)-v\left(d_{U}^{1}\right)\right]+\left(1-p_{L}\right)\left[v\left(b_{H}^{1}\right)-v\left(b_{U}^{1}\right)\right] \\
= & d\left(1-p_{L}\right) p_{L}\left(\alpha_{H}-\alpha_{U}\right)\left[v^{\prime}(\varpi)-v^{\prime}(\nu)\right] .
\end{aligned}
$$


As $\alpha_{H}>\alpha_{U}$ (Lemma 9) and $\varpi<\nu$, then the concavity of $v($.$) implies that$ $\Psi^{M H}\left(c_{\min }, \bar{\Delta}\right)<0$.

Moreover, Result 17 has shown that $\partial \Psi^{M H}(c, \Delta) / \partial c<0$ for $c_{\min }^{M H}<c<c_{\max }^{M H}$. As $\Psi^{M H}(c, \bar{\Delta})$ is continuous in $c$, the intermediate value theorem implies that there exist $\left.\tilde{c}_{2}^{M H}(\bar{\Delta}) \in\right] c_{\min }^{M H}, c_{\max }^{M H}\left[\right.$ such that $\Psi^{M H}(\tilde{c}(\bar{\Delta}), \bar{\Delta})=0$. By continuity of $\Psi^{M H}(c, \Delta)$ in $\Delta$, this threshold $\tilde{c}_{2}^{M H}(\Delta)$ also exists for value of $\Delta$ close enough to $\bar{\Delta}$. Observe that, when it exists, $\tilde{c}_{2}^{M H}(\Delta)<c_{\max }^{M H}$ since $\Psi^{M H}\left(c_{\max }^{M H}, \Delta\right)<0$ for all $\Delta$. From now on, we consider only values of $\Delta$ large enough that $\tilde{c}_{2}^{M H}(\Delta)$ exists.

b) The claim is straightforward since $\partial \Psi^{M H}(c, \Delta) / \partial c<0$ by Result 17 .

c) We have by definition that $\Psi^{M H}\left(\tilde{c}_{2}^{M H}(\Delta), \Delta\right)=0$ so that

$$
\frac{d \Psi^{M H}\left(\tilde{c}_{2}^{M H}(\Delta), \Delta\right)}{d \Delta}=\frac{\partial \Psi^{M H}\left(\tilde{c}_{2}^{M H}(\Delta), \Delta\right)}{\partial c} \frac{\partial \tilde{c}_{2}^{M H}(\Delta)}{\partial \Delta}+\frac{\partial \Psi^{M H}\left(\tilde{c}_{2}^{M H}(\Delta), \Delta\right)}{\partial \Delta}=0 .
$$

Our claim then results from the fact that $\partial \Psi^{M H}(c, \Delta) / \partial c<0$ and that $\partial \Psi^{M H}(c, \Delta) / \partial \Delta>$ 0 for all $c$ and $\Delta$.

\subsection{Proof of Lemma 3}

Proof. Result 6 has shown that $\tilde{c}_{1}(\Delta)<c_{\text {min }}$ exists if $\Delta \geq \tilde{\Delta}\left(\right.$ defined as $\Psi^{M H}\left(c_{\text {min }}, \tilde{\Delta}\right)=$ $0)$, with $\Psi(c, \Delta)<0$ for $c<\tilde{c}_{1}(\Delta)$. Obviously, $\Delta \rightarrow \bar{\Delta}>\tilde{\Delta}$, so that $\tilde{c}_{1}(\Delta)$ exists. Similarly, Result 16 shows that $\tilde{c}_{1}^{M H}(\Delta)$ exists for $\Delta \rightarrow \bar{\Delta}$, with $\Psi^{M H}\left(\tilde{c}_{1}^{M H}(\Delta), \Delta\right)=0$. We then have that $\Psi^{M H}(c, \Delta)>\Psi(c, \Delta)$ for $c<c_{\min }^{M H}$ (Result 15) implies that $\tilde{c}_{1}^{M H}(\Delta)<$ $\tilde{c}_{1}(\Delta)$.

Result 8 has shown that $c_{\min }<\tilde{c}_{2}(\Delta)<c_{\max }$ exists if $\Delta \geq \tilde{\Delta}$, with $\Psi(c, \Delta)>0$ for $\tilde{c}_{1}(\Delta)<c<\tilde{c}_{2}(\Delta)$. Similarly, Result 18 shows that $\tilde{c}_{2}^{M H}(\Delta)<c_{\max }^{M H}$ exists for $\Delta \rightarrow \bar{\Delta}$, with $\Psi^{M H}(c, \Delta)>0$ for $\tilde{c}_{1}^{M H}(\Delta)<c<\tilde{c}_{2}^{M H}(\Delta)$. We then have that $\Psi^{M H}(c, \Delta)<\Psi(c, \Delta)$ for any $\Delta$ when $c_{\min }^{M H}<c_{\min }<c<c_{\max }^{M H}$ (Lemma 4) implies that $\tilde{c}_{2}^{M H}(\Delta)<\tilde{c}_{2}(\Delta)$.

\subsection{Proof of Proposition 5}

We first prove the following lemma.

Lemma $4 \Psi^{M H}(c, \Delta)<\Psi(c, \Delta)$ for any $\Delta$ when $c_{\min }^{M H}<c_{\min }<c<c_{\max }^{M H}$.

Proof. Recall that, when $c_{\min }<c<c_{\max }^{M H}$, we have

$$
\begin{aligned}
\Psi^{M H}(c, \Delta) & =\lambda\left[\left(1-p_{H}^{1}\right) v\left(b_{H}^{1}\right)+p_{H}^{1} v\left(d_{H}^{1}\right)-c\right]+(1-\lambda) v\left(c_{L}\right)-v\left(c_{U}^{0}\right) \\
\Psi(c, \Delta) & =\lambda\left[v\left(c_{H}^{1}\right)-1\right]+(1-\lambda) v\left(c_{L}\right)-v\left(c_{U}^{0}\right)
\end{aligned}
$$


hence we obtain

$$
\Psi(c, \Delta)-\Psi^{M H}(c, \Delta)=\lambda\left[v\left(c_{H}^{1}\right)-\left(1-p_{H}^{1}\right) v\left(b_{H}^{1}\right)-p_{H}^{1} v\left(d_{H}^{1}\right)\right]>0 .
$$

We now prove Proposition 5

Proof. (a) Recall that, when $\Delta$ is close enough to $\bar{\Delta}$, we have that $\Psi^{M H}(0, \Delta)=$ $\Psi(0, \Delta)$ (see Result 14) and that $\Psi^{M H}(c, \Delta)>\Psi(c, \Delta)$ for $c<c_{\min }^{M H}$ (Result 15), which implies that $\Psi^{M H}\left(c_{\text {min }}^{M H}, \Delta\right)>\Psi\left(c_{\text {min }}^{M H}, \Delta\right)$. Lemma 4 shows that $\Psi^{M H}(c, \Delta)<\Psi(c, \Delta)$ for $c_{\min }^{M H}<c_{\min }<c<c_{\max }^{M H}$. By continuity of $\Psi^{M H}(c, \Delta)$ and $\Psi(c, \Delta)$ in $c$, the fact that $\partial \Psi^{M H}(c, \Delta) / \partial c<0$ for $c_{\text {min }}^{M H}<c<c_{\text {min }}$ (see part b) of Result 17) and the intermediate value theorem, we then have that there exists a unique value of $c$, denoted by $\hat{c}$, with $c_{\min }^{M H}<\hat{c}<c_{\text {min }}$, and such that $\Psi^{M H}(c, \Delta)>\Psi(c, \Delta)$ for $c<\hat{c}, \Psi^{M H}(\hat{c}, \Delta)=\Psi(\hat{c}, \Delta)$ and $\Psi^{M H}(c, \Delta)<\Psi(c, \Delta)$ for $\hat{c}<c<c_{\max }$. As for the latter inequality, observe that $\Psi^{M H}(c, \Delta)=\Psi_{0}<\Psi(c, \Delta)$ for $c_{\max }^{M H} \leq c<c_{\max }$, while $\Psi^{M H}(c, \Delta)=\Psi(c, \Delta)=\Psi_{0}$ for $c \geq c_{\max }$.

(b) The proof of Lemma 3 shows that $\tilde{c}_{1}(\Delta), \tilde{c}_{1}^{M H}(\Delta)$ and $\tilde{c}_{2}^{M H}(\Delta)$ exist when $\Delta \rightarrow \bar{\Delta}$. The claim follows from the observation that $\Psi^{M H}(c, \Delta)>0$ for $\tilde{c}_{1}^{M H}(\Delta)<c<\tilde{c}_{2}^{M H}(\Delta)$ (Results 16 and 18) while $\Psi(c, \Delta)<0$ for for $c<\tilde{c}_{1}(\Delta)$ (Result 6 ).

(c) The proof of Lemma 3 shows that $\tilde{c}_{1}(\Delta), \tilde{c}_{2}(\Delta)$ and $\tilde{c}_{2}^{M H}(\Delta)$ exist when $\Delta \rightarrow \bar{\Delta}$. The claim follows from the observation that $\Psi^{M H}(c, \Delta)<0$ for $c>\tilde{c}_{2}^{M H}(\Delta)$ (Result 3) while $\Psi(c, \Delta)>0$ for $\tilde{c}_{1}(\Delta)<c<\tilde{c}_{2}(\Delta)$ (Result 1 (b)).

(d) We know that $\Psi(c, \Delta)$ is maximized at $c=c_{\text {min }}$, and we now show that from $\Psi^{M H}\left(c_{\text {min }}^{M H}, \Delta\right)>\Psi\left(c_{\text {min }}, \Delta\right)$. We have that

$$
\begin{aligned}
\Psi^{M H}\left(c_{\text {min }}^{M H}, \Delta\right) & =\lambda\left[\left(1-p_{H}^{1}\right) v\left(b_{H}^{1}\right)+p_{H}^{1} v\left(d_{H}^{1}\right)-\left(1-p_{U}^{1}\right) v\left(b_{U}^{1}\right)-p_{U}^{1} v\left(d_{U}^{1}\right)\right]+(1-\lambda)\left[v\left(c_{L}\right)-v\left(c_{U}^{0}\right)\right], \\
\Psi\left(c_{m i n}, \Delta\right) & =\lambda\left[v\left(c_{H}^{1}\right)-v\left(c_{U}^{1}\right)\right]+(1-\lambda)\left[v\left(c_{L}\right)-v\left(c_{U}^{0}\right)\right],
\end{aligned}
$$

so that

$$
\begin{aligned}
& \Psi^{M H}\left(c_{\text {min }}^{M H}, \Delta\right)>\Psi\left(c_{\text {min }}, \Delta\right) \\
\Leftrightarrow & v\left(c_{U}^{1}\right)-\left[\left(1-p_{U}^{1}\right) v\left(b_{U}^{1}\right)-p_{U}^{1} v\left(d_{U}^{1}\right)\right]>v\left(c_{H}^{1}\right)-\left[\left(1-p_{H}^{1}\right) v\left(b_{H}^{1}\right)+p_{H}^{1} v\left(d_{H}^{1}\right)\right] .
\end{aligned}
$$

If we assume that $\Delta=\bar{\Delta}$, the latter inequality becomes

$$
\begin{aligned}
\left(1-p_{L}\right)\left[v\left(b_{U}^{1}\right)-v\left(b_{H}^{1}\right)\right] & <p_{L}\left[v\left(d_{H}^{1}\right)-v\left(d_{U}^{1}\right)\right] \\
\left(1-p_{L}\right)\left[b_{U}^{1}-b_{H}^{1}\right] v^{\prime}(\alpha) & <p_{L}\left[d_{H}^{1}-d_{U}^{1}\right] v^{\prime}(\beta),
\end{aligned}
$$

with $\alpha>\beta$. Using

$$
\begin{aligned}
& b_{U}^{1}-b_{H}^{1}=\left(\alpha_{H}-\alpha_{U}\right) p_{L} d \\
& b_{U}^{1}-b_{H}^{1}=\left(\alpha_{H}-\alpha_{U}\right) p_{L} d
\end{aligned}
$$


the inequality (13) becomes

$$
v^{\prime}(\alpha)<v^{\prime}(\beta)
$$

which is true.

By continuity of $\Psi^{M H}\left(c_{m i n}^{M H}, \Delta\right)$ and of $\Psi\left(c_{m i n}, \Delta\right)$ in $\Delta$, we obtain that $\Psi^{M H}\left(c_{m i n}^{M H}, \Delta\right)>$ $\Psi\left(c_{\min }, \Delta\right)$ for $\Delta \rightarrow \bar{\Delta}$. 
Figure 1: Value of test as a function of $c$ for several values of $\Delta$

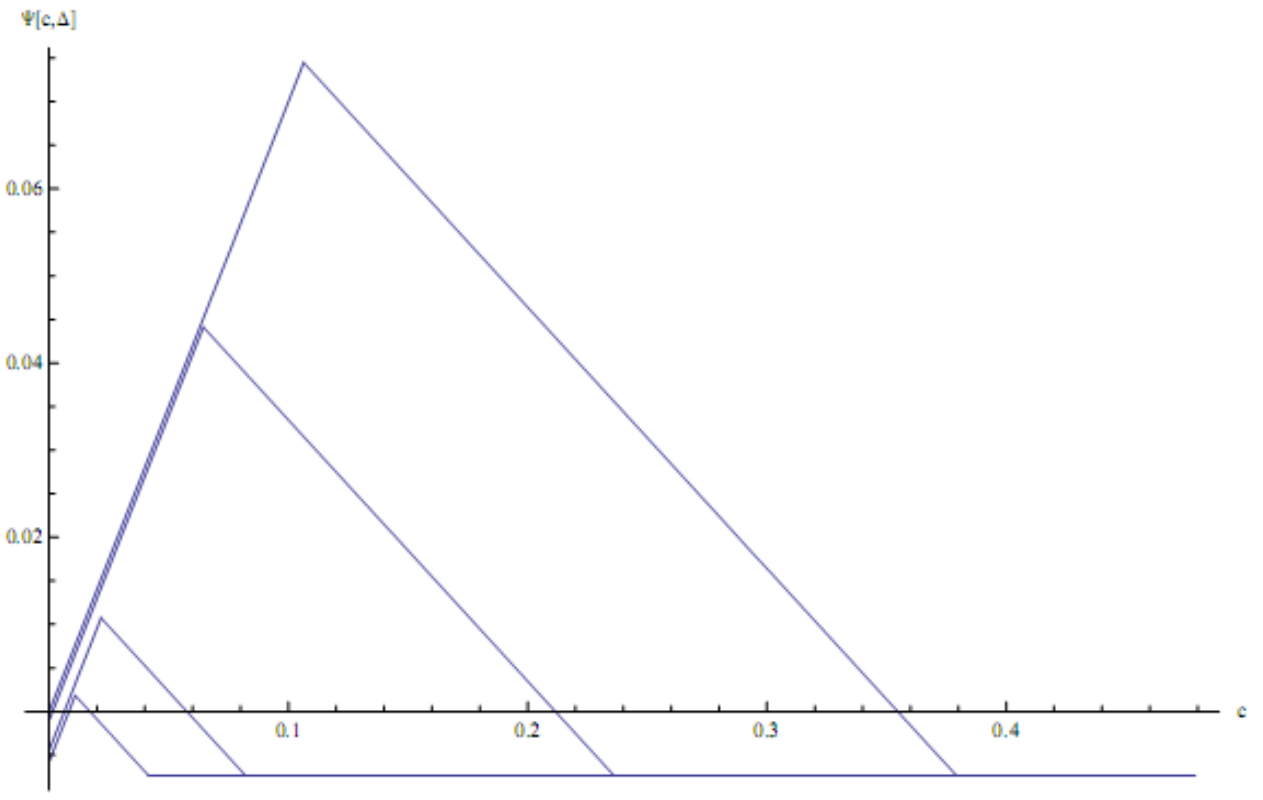


Figure 2 : Value of $c-1$ (yellow), cmin (blue), c -2 (green) and cmax (purple) as a function of $\Delta$ cilda, cmin \& cmax

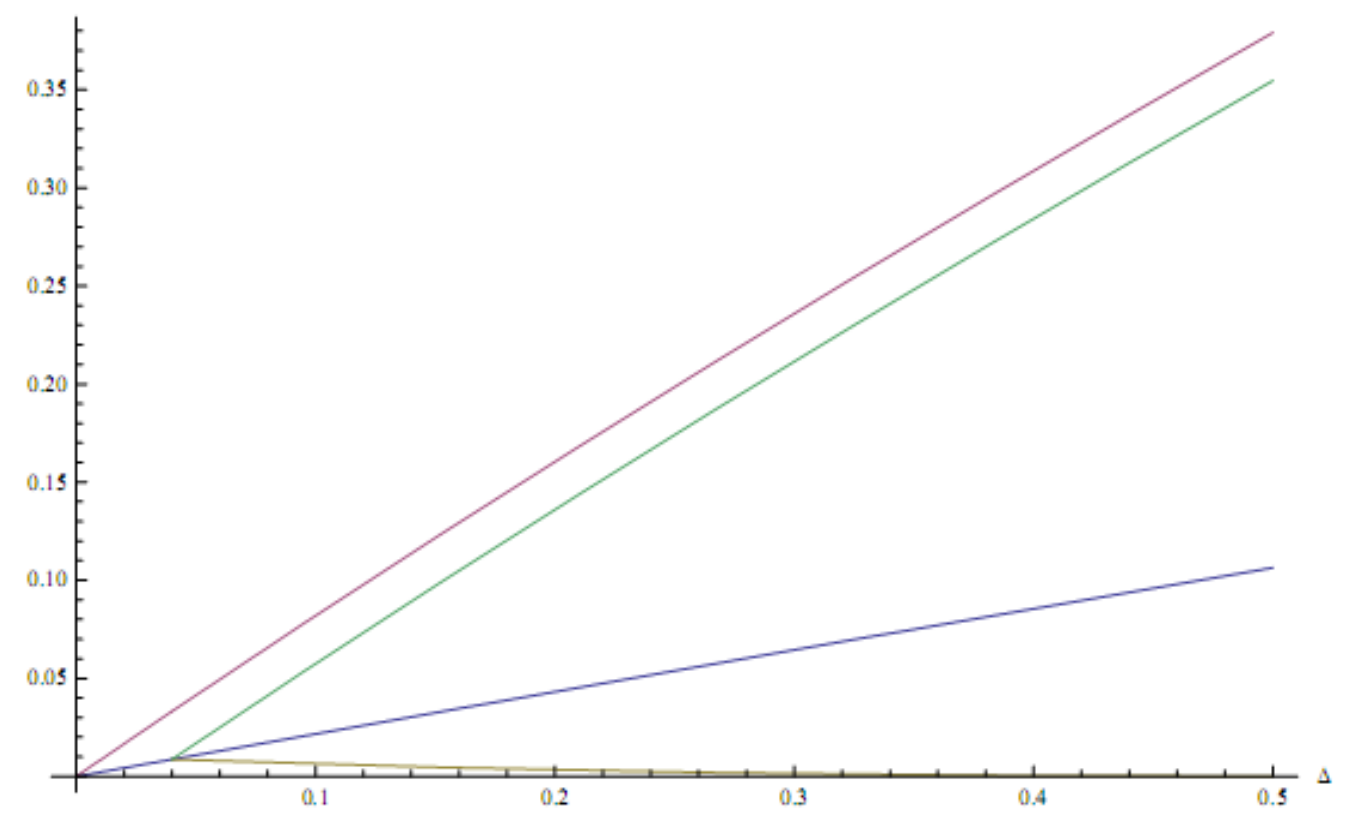


Figure 3: $\alpha \mathrm{H}$ and $\alpha \mathrm{U}(\alpha \mathrm{U}<\alpha \mathrm{H})$ as a function of $\mathrm{c}$

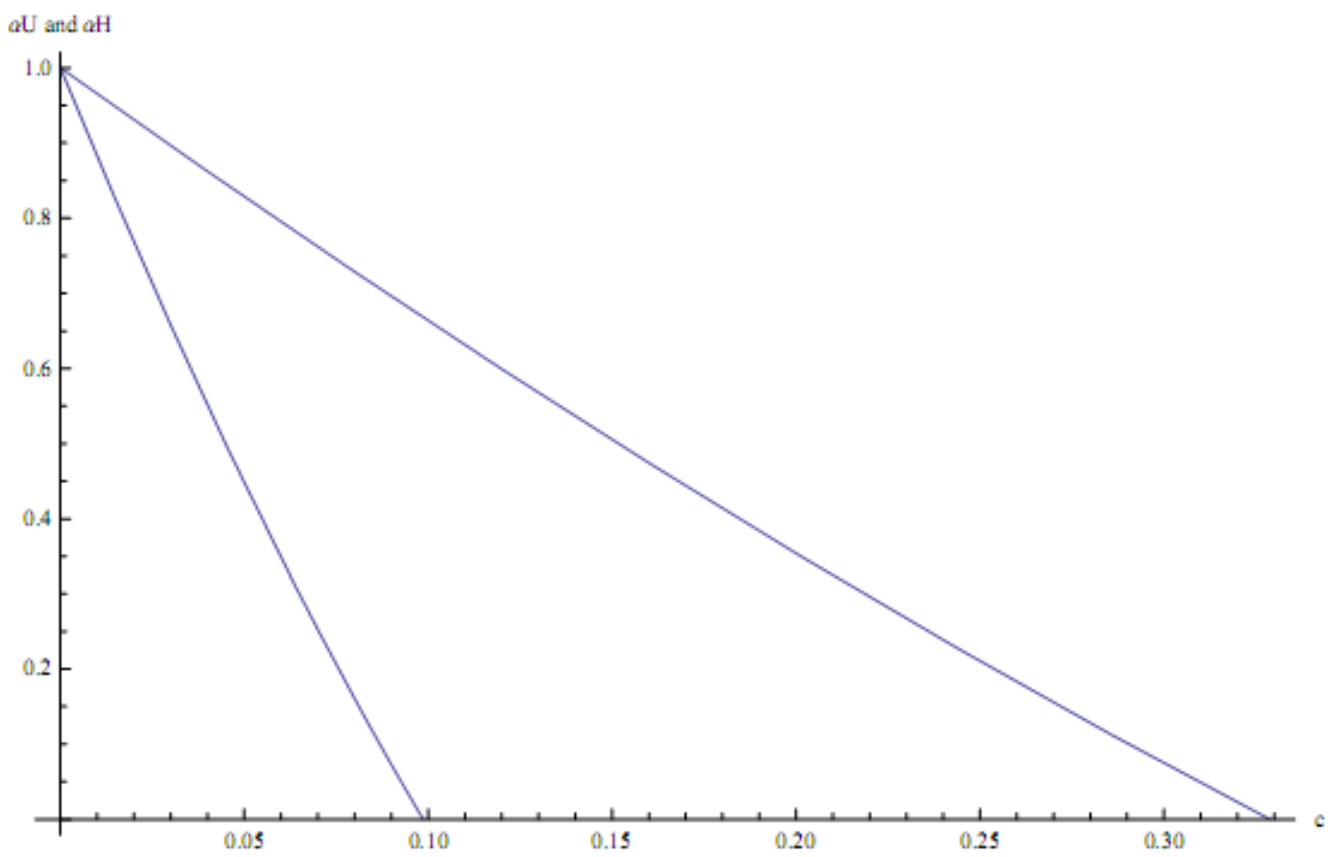




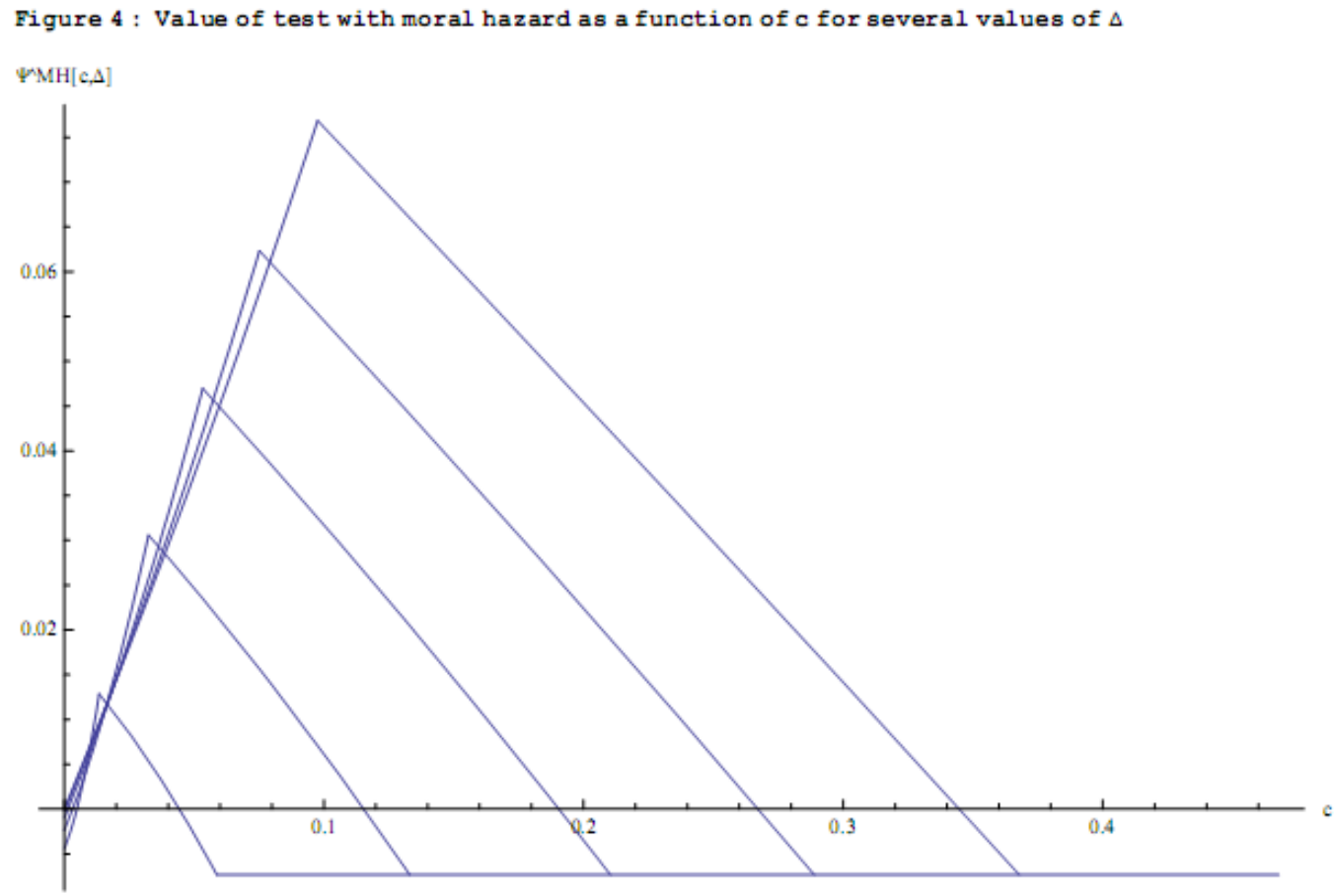


Figure 5: Value of test with moral hazard (zoom of Figure 4 on low values of c)

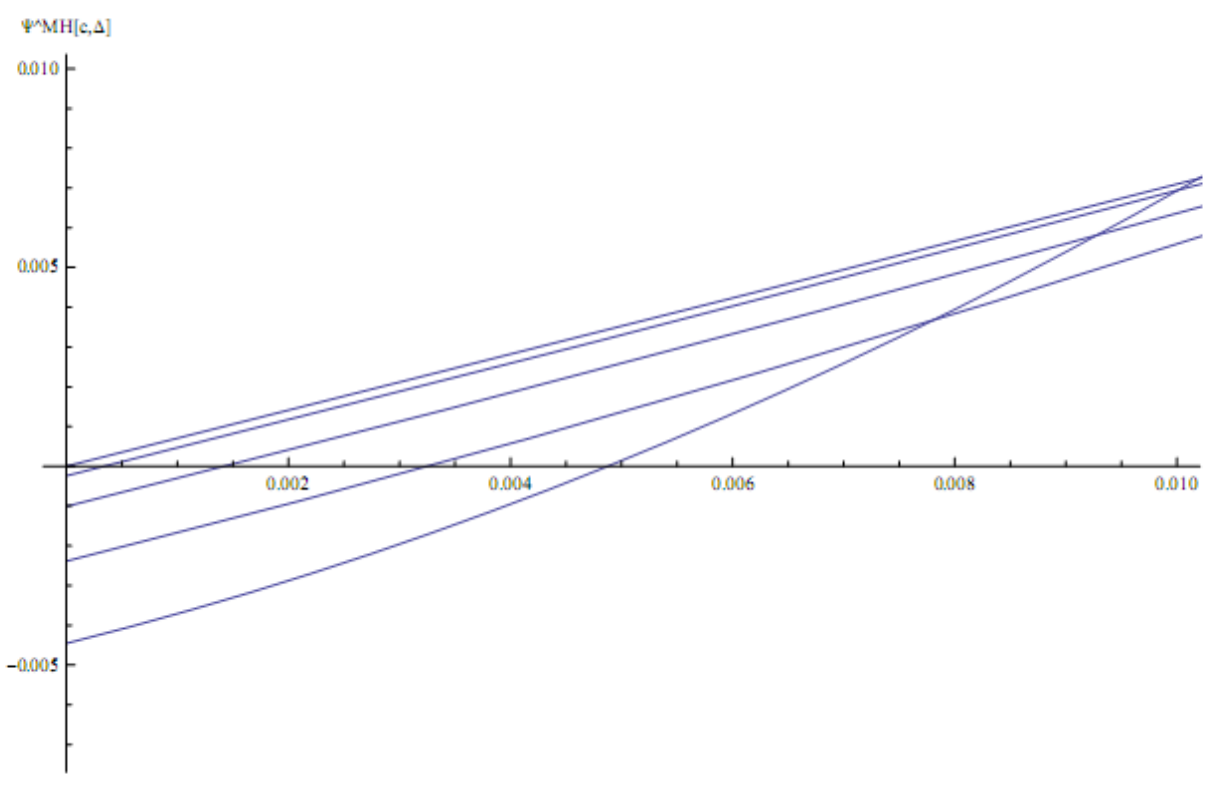


Figure 6 : Comparison of value of test,

with (right) and wi thout MH (left), as a function of c for $\Delta=0.1$

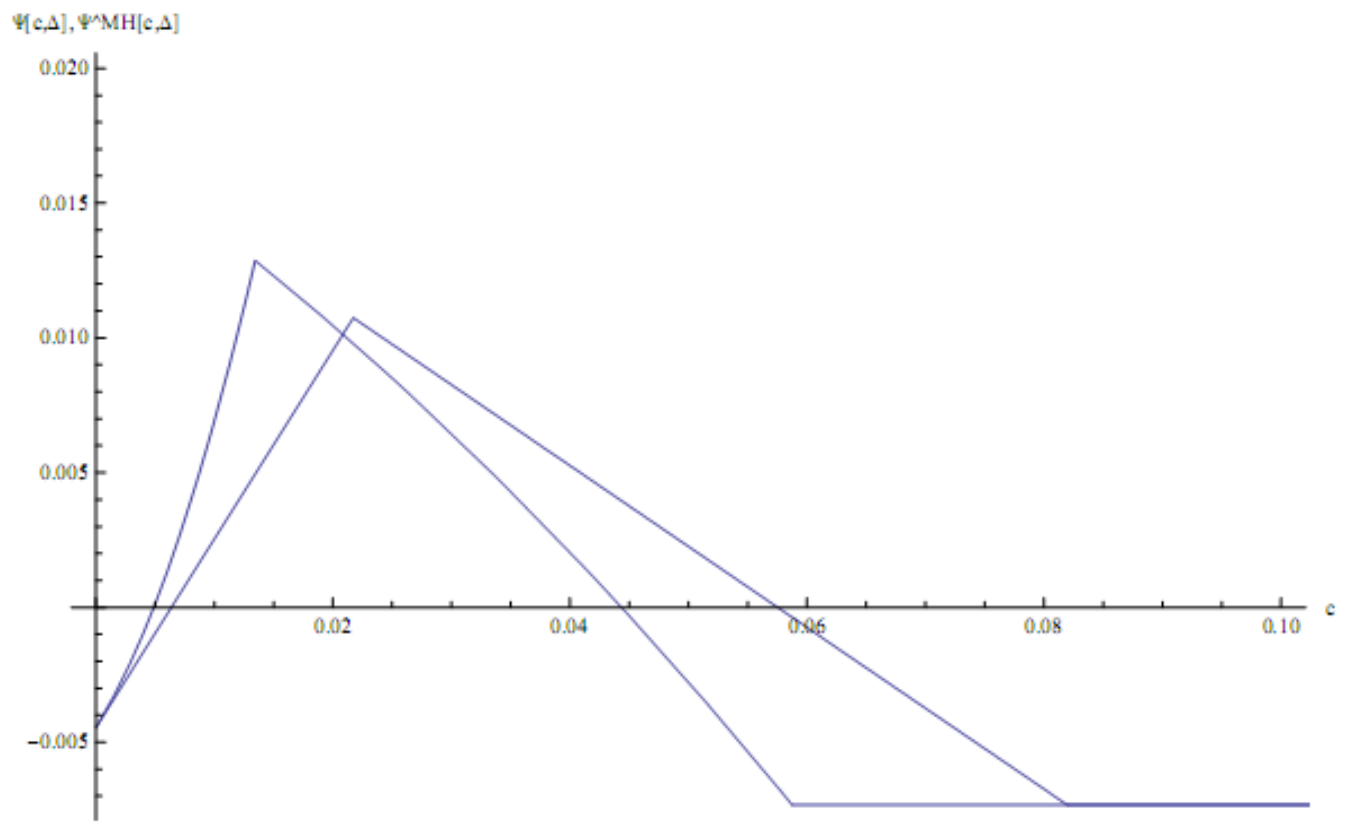


Figure 7: Comparison of $\mathrm{cMH} \sim 1$ and $\mathrm{cMH} \sim 2$ with $\mathrm{c}-1$ and $\mathrm{c}-2$, as a function of $\Delta$

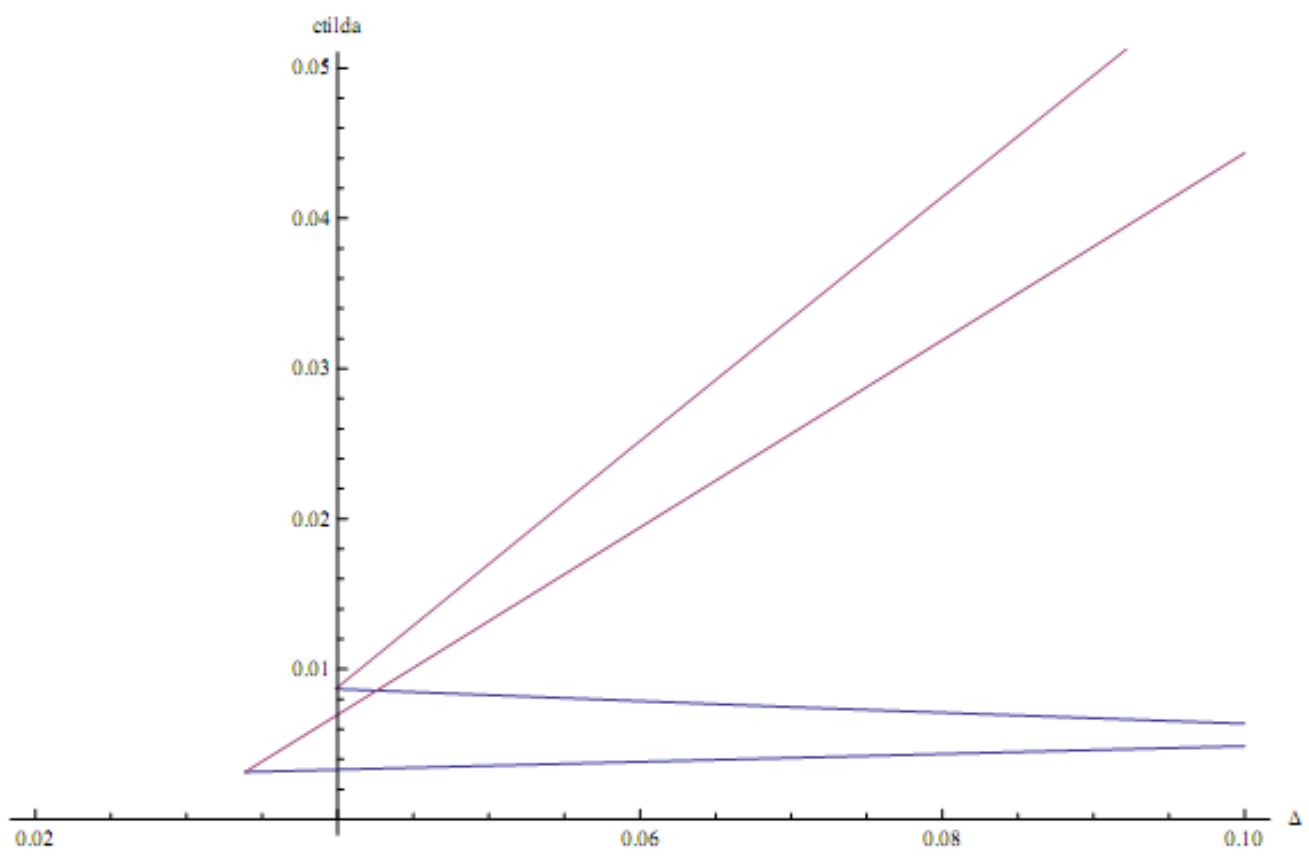


Figure 8 : Ex-ante utility as a function of effort cost

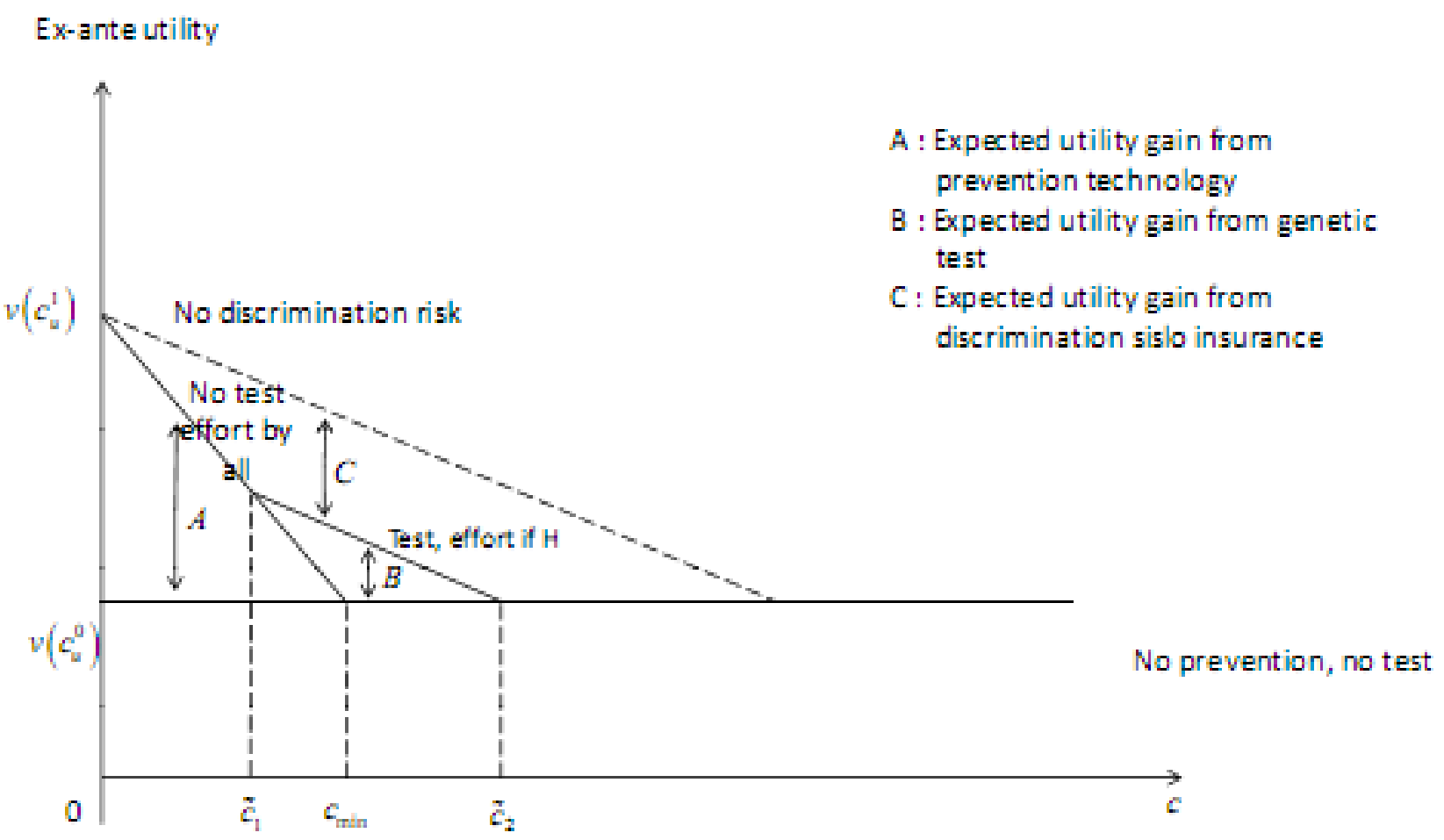


Figure 9 : Ex-ante utility with and without mora I hazard

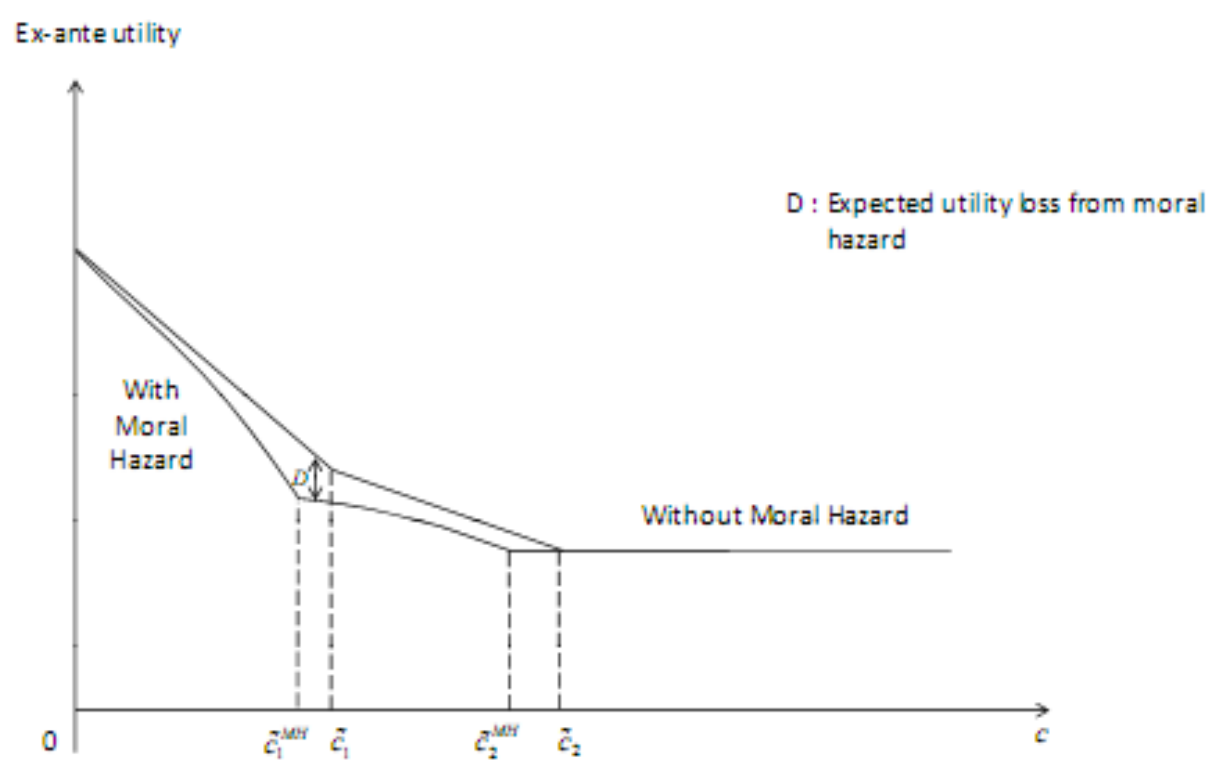

